\title{
Synthesis, characterization, molecular docking evaluation, antiplatelet and anticoagulant actions of 1,2,4 triazole hydrazone and sulphonamide novel derivatives
}

\author{
Waseem Khalid ${ }^{1}$, Amir Badshah ${ }^{1}$, Arif-ullah Khan ${ }^{1 *}$, Humaira Nadeem $^{1}$ and Sagheer Ahmed ${ }^{2}$
}

\begin{abstract}
In the present study, a series of new hydrazone and sulfonamide derivatives of 1,2,4-triazole were synthesized. Initially three 4-substituted-5-(2-pyridyl)-1,2,4-triazole-3-thiones ZE-1 (a-c) were treated with ethyl chloroacetate to get the corresponding thioesters ZE-2(a-c), which were reacted with hydrazine hydrate to the respective hydrazides ZE-3(ac). The synthesized hydrazides were condensed with different aldehydes and p-toluene sulfonylchloride to furnish the target hydrazone derivatives $\mathrm{ZE}-4(\mathrm{a}-\mathrm{c})$ and sulfonamide derivatives $\mathrm{ZE}-5(\mathrm{a}-\mathrm{c})$ respectively. All the synthesized compounds were characterized by FTIR, ${ }^{1} \mathrm{HNMR},{ }^{13} \mathrm{CNMR}$ and elemental analysis data. Furthermore, the new hydrazone and sulfonamide derivatives ZE-4(b-c) and ZE-5(a-b) were evaluated for their antiplatelet and anticoagulant activities. ZE-4b, ZE-4c, ZE-5a and ZE-5b inhibited arachidonic acid, adenosine diphosphate and collagen-induced platelets aggregation with IC ${ }_{50}$ values of 40.1, 785 and 10.01 (ZE-4b), 55.3, 850.4 and 10 (ZE-4C), 121.6, 956.8 and 30.1 (ZE-5a), 99.9, 519 and 29.97 (ZE-5b) respectively. Test compounds increased plasma recalcification time (PRT) and bleeding time (BT) with ZE-4c being found most effective, which at 30, 100, 300 and $1000 \mu \mathrm{M}$ increased PRT to $84.2 \pm 1.88$, $142 \pm 3.51,205.6 \pm 5.37$ and $300.2 \pm 3.48 s$ and prolonged BT to $90.5 \pm 3.12,112.25 \pm 2.66,145.75 \pm 1.60 \mathrm{~s}(\mathrm{P}<0.001$ vs. saline group) respectively. In silico docking approach was also applied to screen these compounds for their efficacy against selected drug targets of platelet aggregation and blood coagulation. Thus in silico, in vitro and in vivo investigations of ZE-4b, ZE-4c, ZE-5a and ZE-5b prove their antiplatelet and anticoagulant potential and can be used as lead molecules for further development.
\end{abstract}

Keywords: 1,2,4-Triazole derivatives, Hydrazone and sulphonamide derivatives, Antiplatelet, Anticoagulant

\section{Introduction}

Thrombotic disorders are responsible for major health problems worldwide [1]. According to global burden of diseases, injuries and risk factors study, ischemic heart diseases caused 7.0 million deaths and stroke up to 5.9 million deaths in 2010 only. About $50 \%$ of these deaths were caused by thrombosis [2]. Hemostasis maintains normal blood flow in our body and prevents blood loss after vascular injury. Platelet and coagulation factors are

\footnotetext{
*Correspondence: arif.ullah@riphah.edu.pk

${ }^{1}$ Riphah Institute of Pharmaceutical Sciences, Riphah International University, Islamabad, Pakistan

Full list of author information is available at the end of the article
}

essential elements of hemostasis, which are involved in activation and stabilization of thrombin resulting in the formation of thrombus and thus prevention of hemorrhage $[3,4]$. Disturbance in normal hemostatic balance or platelet function contributes to development and progression of many thrombotic disorders [5]. There are many antiplatelet and anticoagulant drugs, available commercially, which are being used for the treatment of thrombotic disorders. But these agents are associated with numerous limitations and side effects, including lack of reversibility, a sheer dose response, interactions, narrow therapeutic index, congenital disabilities, miscarriage and most commonly bleeding complications $[6,7]$. Therefore, identifying target specific novel antiplatelet 
and anticoagulant agents with a better efficacy and least side effects is a challenging task for researchers.

Triazole is a five-membered heterocyclic compound with two isomeric forms, i.e. 1,2,3-triazole and 1,2,4-triazole. 1,2,4-Triazoles especially have received much attention as their intriguing physical and biological properties, as well as their excellent stability, rendering them potential drug core structures. Triazole derivatives have wide pharmacological spectrum such as antimicrobial, anti-inflammatory, analgesic, antimalarial, antiviral, antiproliferative, anticancer and various other activities [8]. In a recent study, 1,2,3-triazole derivatives have also shown significant inhibitory activity against blood platelet aggregation and coagulation [9]. Hydrazone is a class of organic compounds having azomethine group $\mathrm{R}_{1} \mathrm{R}_{2} \mathrm{C}=\mathrm{NNH}_{2}$, which are known to possess different pharmacological activities like antimicrobial, analgesic, anti-inflammatory, anticonvulsant, antidiabetic, antitumor and antiplatelet activities [10]. Similarly, sulfonamides are well known class of compounds associated with broad range of activities including antibacterial, anti-inflammatory, carbonic anhydrase inhibitor, hypoglycemic activity, anti-HIV, anticancer and antiplatelet activities [11]. In view of the great importance of triazole, hydrazone and sulfonamide moieties in medicinal chemistry, we would like to report the synthesis of some new hydrazone and sulfonamide derivatives of 4,5-disubstituted-1,2,4-triazole-3-thiones ZE-4(a-c) and $\mathrm{ZE}-5(\mathrm{a}-\mathrm{c})$. ZE is the structural code given to the synthesized compounds. The synthesized derivatives $\mathrm{ZE}-4(\mathrm{~b}-\mathrm{c})$ and ZE-5(a-b), as shown in Fig. 1, were investigated for their antiplatelet and anticoagulant effects using in vitro and in vivo assays. In addition to this, molecular docking study of synthesized compounds was also performed against selected targets of platelet aggregation and blood coagulation pathways to study the binding interactions which can provide an insight into the possible mechanism of action of these new molecules.

\section{Materials and methods Chemicals}

Benzaldehyde, dimethyl sulfoxide, ethanol, ethyl chloroacetate, potassium hydroxide $(\mathrm{KOH}), p$-toluene-sulphonyl-chloride were obtained from Merck Millipore., Billerica, MA, USA. Aspirin, calcium chloride $\left(\mathrm{CaCl}_{2}\right)$, diethyl ether, heparin, phosphate buffers solution (PBS), sodium citrate from Sigma chemicals., Dt. Louis, MO, USA. Adenosine diphosphate (ADP), arachidonic acid (AA) and collagen were purchased from Chrono-log association, Havertown, PA, USA.

\section{Animals}

Balb-C mice (25-30 g) of either sex were used, housed at animal house of Riphah Institute of Pharmaceutical
Sciences (RIPS) under standard laboratory protocols; at $25 \pm 2{ }^{\circ} \mathrm{C}$, duration of light and darkness was set for $12 \mathrm{~h}$ each. Mice were given free access to standard diet and water ad libitum. The study performed complied with rules of Institute of Laboratory Animal Resources, Commission on Life Sciences University, National Research Council (1996), approved by RIPS Ethical Committee (Reference No: REC/RIPS/2016/008).

\section{Chemistry}

All chemicals were purchased from commercial suppliers and used without further purification. Melting points were determined on a Gallenkamp melting point apparatus and were uncorrected. The IR spectra were recorded on Thermo scientific NICOLET IS10 spectrophotometer. All ${ }^{1} \mathrm{HNMR}$ and ${ }^{13} \mathrm{CNMR}$ spectra were recorded on Bruker AM-400 spectrophotometer at 400 and $100 \mathrm{MHz}$ respectively, in DMSO as a solvent and TMS as an internal standard. Elemental analyses were performed with a LECO-183 CHN analyzer. 1,2,4-Triazole hydrazone and sulphonamide derivatives were synthesized in three steps, following Scheme 1.

\section{Synthesis of 5-(substituted)-1,2,4-triazole-2-thiones ZE-1(a-c)}

All the substituted mercapto triazoles $\mathrm{ZE}-1(\mathrm{a}-\mathrm{c})$ were synthesized previously by the reported procedure. The triazoles were characterized by comparing their melting points with the reported literature [12].

\section{Synthesis of 1,2,4-triazole esters ZE-2(a-c)}

$0.003 \mathrm{~mol}$ of respective triazoles $\mathrm{ZE}-1(\mathrm{a}-\mathrm{c})$ were dissolved in $50 \mathrm{~mL}$ of absolute ethanol and a solution of $0.003 \mathrm{~mol}(0.168 \mathrm{~g})$ of $\mathrm{KOH}$ in $20 \mathrm{~mL}$ of water was added dropwise to the mixture with continuous stirring. After 30-min, ethyl chloroacetate was slowly added to the reaction mixture and refluxed for $2-3 \mathrm{~h}$. The progress of the reaction was monitored by thin layer chromatography (TLC) (ethyl acetate: petroleum ether 2:1). After completion of the reaction, the solvent was evaporated in vacuo and the crude product thus obtained was recrystallized from ethanol to get the corresponding triazole thioesters ZE-2(a-c) $[12,13]$.

Ethyl [\{4-cyclohexyl-5-(pyridine-2-yl)-4H-1,2,4-triazol-3-yl]sulfanyl\}acetate (ZE-2a) Yield 78\%, M.P. 147$149{ }^{\circ} \mathrm{C}, \mathrm{R}_{\mathrm{f}} 0.77$ (ethyl acetate: pet. ether 2:1); IR (KBr) $\mathrm{cm}^{-1}: 2972(\mathrm{C}-\mathrm{H}), 1726(\mathrm{C}=\mathrm{O}$, ester $), 1665(\mathrm{C}=\mathrm{N})$, $1505(\mathrm{C}=\mathrm{C}) ;{ }^{1} \mathrm{H}-\mathrm{NMR}\left(\mathrm{DMSO}-\mathrm{d}_{6}, 400 \mathrm{MHz}\right): \delta 8.60(\mathrm{~d}$, $1 \mathrm{H}, \mathrm{J}=7.6 \mathrm{~Hz}, \mathrm{Py} \mathrm{H}-3), 8.01$ (d, $1 \mathrm{H}, \mathrm{J}=7.9$, Py H-6), $7.80(\mathrm{t}, 1 \mathrm{H}, \mathrm{J}=7.8 \mathrm{~Hz}$, Py H-4), $7.36(\mathrm{dd}, 1 \mathrm{H}, \mathrm{J}=7.6 \mathrm{~Hz}$, $\mathrm{J}=7.8 \mathrm{~Hz}$, Py H-5), 4.45 (m, 1H, cyclohexyl H-1), 4.12 (s, 2H, $\mathrm{CH}_{2}-\mathrm{S}$ ), $3.16\left(\mathrm{q}, 2 \mathrm{H}, \mathrm{J}=7.0 \mathrm{~Hz}, \mathrm{OCH}_{2}\right), 1.31$ (t, 
ZE-4b<smiles>CCn1c(SCC(=O)N/N=C/c2ccccc2)nnc1-c1ccccn1</smiles>

ZE-5a

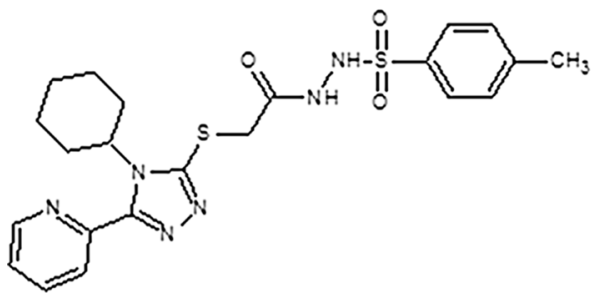

ZE-4c<smiles>O=C(CSc1nnc(-c2ccccn2)n1-c1ccc(F)cc1)N/N=C/c1ccccc1</smiles>

ZE-5b<smiles>CCn1c(SCC(=O)NNS(=O)(=O)c2ccc(C)cc2)nnc1-c1ccccn1</smiles>

Fig. 1 Structures of compounds: N-\{\{(2-phenyl)methylidene]-2-(4-ethyl-5-(pyridine-2-yl)-4H-1,2,4-triazole-3-yl)sulfanyl\}acetohydrazide (ZE-4b), $\mathrm{N}$-[\{(2-phenyl)methylidene]-2-(4-(fluorophenyl-5-(pyridine-2-yl)-4H-1,2,4-triazole-3yl)sulfanyl\}acetohydrazide (ZE-4c), $N$-[\{(4-methylphenyl)sulfonyl\}]2-(4-cyclohexyl-5-(pyridine-2-yl)-4H-1,2,4-triazole-3-yl)sulfanyl\}acetohydrazide (ZE-5a) and N-\{((4-methylphenyl)sulfonyl\}-2-(4-ethyl-5-(pyridine-2-yl)$4 H-1,2,4$-triazole-3yl)sulfanyl\}acetohydrazide (ZE-5b)

$\left.3 \mathrm{H}, \mathrm{J}=6.9 \mathrm{~Hz}, \mathrm{CH}_{3}\right), 1.25-1.81(\mathrm{~m}, 10 \mathrm{H}$, cyclohexyl $\mathrm{H})$. ${ }^{13}$ CNMR (DMSO-d, $\left.100 \mathrm{MHz}\right): \delta 167.8(\mathrm{C}=\mathrm{O}), 152.5$, 146.3, 145.6, 143.2, 135.4, 123.3, 120.4, 62.1, 58.3, 57.2, 30.6, 29.8 (2C), 25.4 (2C), 24.9, 13.8. Anal. Calcd. For $\mathrm{C}_{17} \mathrm{H}_{22} \mathrm{~N}_{4} \mathrm{O}_{2} \mathrm{~S}: \mathrm{C}, 58.95 ; \mathrm{H}, 6.35 ; \mathrm{N}, 16.18$.

Found: C, 58.56; H, 6.40; N, 16.27.

Ethyl [\{4-ethyl-5-(pyridine-2-yl)-4H-1,2,4-triazol-3-yl] sulfanyl\}acetate (ZE-2b) Yield 81\%, M.P. $155-157{ }^{\circ} \mathrm{C}$, $\mathrm{R}_{\mathrm{f}} 0.81$ (ethyl acetate: petroleum ether 2:1); IR (KBr) $\mathrm{cm}^{-1}$ : $2985(\mathrm{C}-\mathrm{H}), 1730(\mathrm{C}=\mathrm{O}$, ester $), 1625(\mathrm{C}=\mathrm{N}) 1446$ $(\mathrm{C}=\mathrm{C}) ;{ }^{1} \mathrm{HNMR}\left(\mathrm{DMSO}-\mathrm{d}_{6}, 400 \mathrm{MHz}\right): \delta 8.71(\mathrm{~d}, 1 \mathrm{H}$, $\mathrm{J}=7.6 \mathrm{~Hz}$, Py H-3), 8.05 (d, 1H, J = 7.9 Hz, Py H-6), 8.01 $\left(\mathrm{t}, 1 \mathrm{H}, \mathrm{J}=7.6 \mathrm{~Hz}\right.$, Py H-4), $7.41\left(\mathrm{dd}, 1 \mathrm{H}, \mathrm{J}_{4,5}=7.5 \mathrm{~Hz}\right.$, $\mathrm{J}_{5,6}=7.9 \mathrm{~Hz}$, Py H-5), 4.50 (q, 2H, J = 6.9 Hz, $\mathrm{CH}_{2}$ ), 4.29 (s, 2H, $\mathrm{CH}_{2}-\mathrm{S}$ ), 3.67 (q, $2 \mathrm{H}, \mathrm{J}=6.8 \mathrm{~Hz}, \mathrm{OCH}_{2}$ ), 1.33 $\left(\mathrm{t}, 3 \mathrm{H}, \mathrm{J}=7.0 \mathrm{~Hz}, \mathrm{CH}_{3}\right), 1.30\left(\mathrm{t}, 3 \mathrm{H}, \mathrm{J}=6.7 \mathrm{~Hz}, \mathrm{CH}_{3}\right)$. ${ }^{13}$ CNMR (DMSO-d, $\left.100 \mathrm{MHz}\right): \delta 166.7(\mathrm{C}=\mathrm{O}), 153.1$, 147.2, 146.6, 145.4, 134.8, 122.7, 121.3, 61.8, 42.5, 32.5, 13.2, 12.1. Anal. Calcd. For $\mathrm{C}_{13} \mathrm{H}_{16} \mathrm{~N}_{4} \mathrm{O}_{2} \mathrm{~S}: \mathrm{C}, 53.42 ; \mathrm{H}$, 5.47; N, 19.17 .

Found: C, 53.40; H, 5.39; N, 19.10.

Ethyl [\{4-(4-flurophenyl)-5-(pyridine-2-yl)-4H-1,2,4 -triazol-3-yl]sulfanyl\}acetate (ZE-2c) Yield 78\%, M.P. 252-260 ${ }^{\circ} \mathrm{C}, \mathrm{R}_{\mathrm{f}} 0.79$ (ethyl acetate: petroleum ether 2:1);IR (KBr) cm ${ }^{-1}$ : $2985(\mathrm{C}-\mathrm{H}), 1735$ (C=O, ester), 1607
$(\mathrm{C}=\mathrm{N}), 1510(\mathrm{C}=\mathrm{C}) ;{ }^{1} \mathrm{H}-\mathrm{NMR}$ (DMSO-d, $\left.400 \mathrm{MHz}\right): \delta$ $8.39(\mathrm{~d}, 1 \mathrm{H}, \mathrm{J}=7.7 \mathrm{~Hz}$, Py H-3), $8.00(\mathrm{~d}, 1 \mathrm{H}, \mathrm{J}=7.8 \mathrm{~Hz}$, Py H-6), 7.60 (t, 1H, J = 7.6 Hz, Py H-4), 7.36 (dd, 1H, $\mathrm{J}_{4,5}=7.5, \mathrm{~J}_{5,6}=7.6 \mathrm{~Hz}$, Py H-5), 7.26-7.31 (m, 4H, $\mathrm{Ar}-\mathrm{H}), 4.33\left(\mathrm{~s}, 2 \mathrm{H}, \mathrm{CH}_{2}-\mathrm{S}\right), 3.41(\mathrm{q}, 2 \mathrm{H}, \mathrm{J}=6.9 \mathrm{~Hz}$, $\mathrm{OCH}_{2}$ ), $1.27\left(\mathrm{t}, 3 \mathrm{H}, \mathrm{J}=6.7 \mathrm{~Hz}, \mathrm{CH}_{3}\right) .{ }^{13} \mathrm{CNMR}$ (DMSO$\left.\mathrm{d}_{6}, 100 \mathrm{MHz}\right): \delta 166.7(\mathrm{C}=\mathrm{O}), 160.1(\mathrm{C}-\mathrm{F}), 152.6,147.3$, 146.2, 145.0, 143.7, 136.3, 124.8 (2C), 123.6, 122.7, 115.6 (2C), 60.8, 32.6, 13.8. Anal. Calcd. For $\mathrm{C}_{17} \mathrm{H}_{15} \mathrm{~N}_{4} \mathrm{O}_{2} \mathrm{SF}: \mathrm{C}$, 56.98; H, 4.18; N, 15.64 .

Found: C, 56.96; H, 4.15; N, 15.39.

Synthesis of 1,2,4-triazolehydrazides ZE-3(a-c)

A mixture of $0.002 \mathrm{~mol}$ of respective triazole esters $\mathrm{ZE}-2(\mathrm{a}-\mathrm{c})$ and $0.006 \mathrm{~mol}$ of hydrazine hydrate in absolute ethanol was refluxed for $4-5 \mathrm{~h}$ with stirring. The progress of the reaction was monitored by TLC (ethyl acetate: petroleum ether 2:1). After completion, the reaction mixture was allowed to cool and excess hydrazine was evaporated. The crude solid was filtered off and recrystallized from ethanol to give the corresponding hydrazides ZE-3(a-c) [14].

2-[\{4-Cyclohexyl-5-(pyridine-2-yl)-4H-1,2,4-triazol-3-yl] sulfanyl\}acetohydrazide (ZE-3a) Yield 68\%, M.P. 143-145 ${ }^{\circ} \mathrm{C}, \mathrm{R}_{\mathrm{f}} 0.78$ (ethyl acetate: petroleum ether 2:1); IR (KBr) cm cm $^{-1} 3347(\mathrm{~N}-\mathrm{H}), 2985(\mathrm{C}-\mathrm{H}), 1687$ (C=O, 


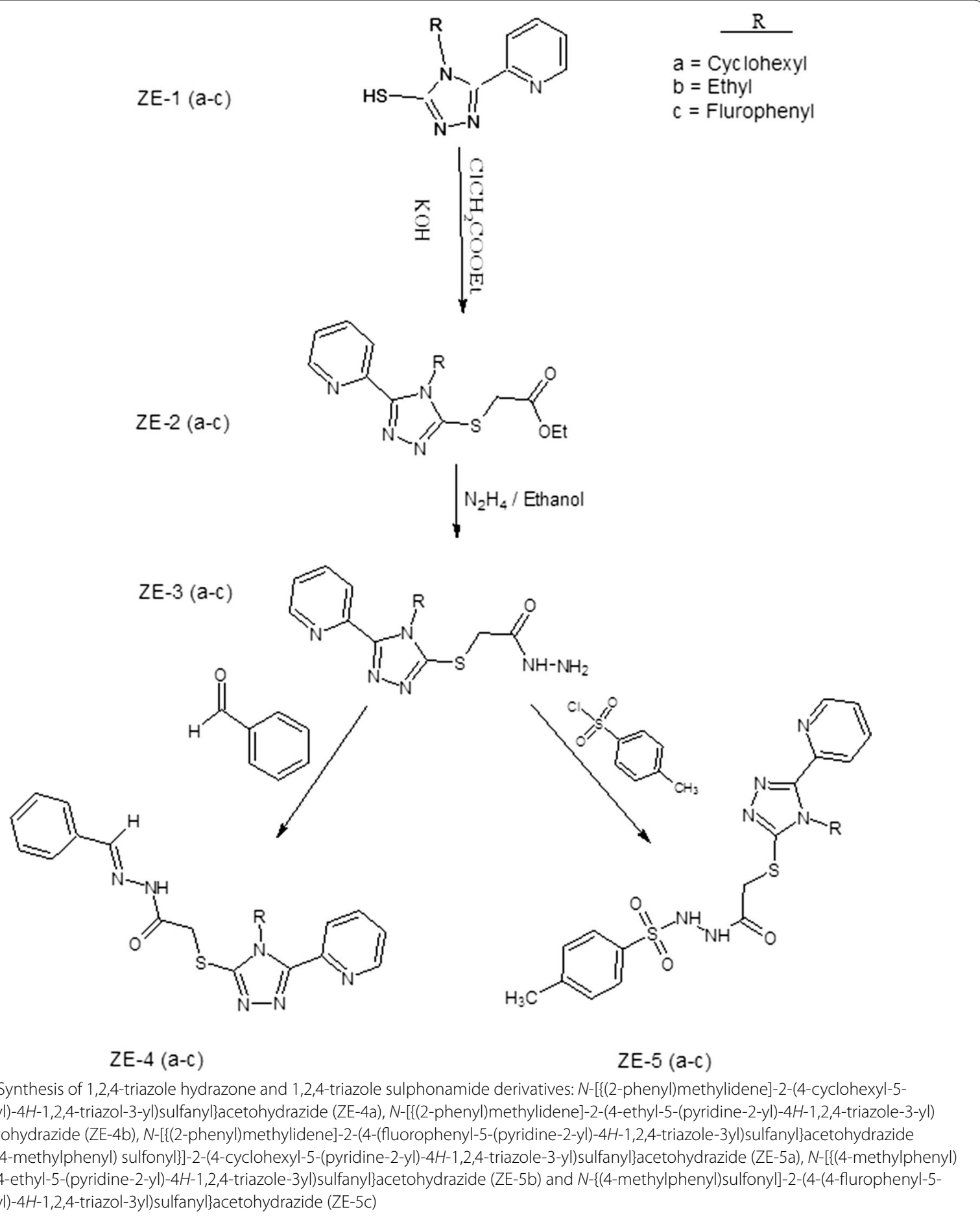

amide), $1650(\mathrm{C}=\mathrm{N}), 1448(\mathrm{C}=\mathrm{C})$; ${ }^{1} \mathrm{HNMR}$ (DMSO- $\mathrm{d}_{6}$, $400 \mathrm{MHz}): \delta 9.23(\mathrm{~s}, 1 \mathrm{H}, \mathrm{NH}), 8.75(\mathrm{~d}, 1 \mathrm{H}, \mathrm{J}=7.4 \mathrm{~Hz}, \mathrm{Py}$ $\mathrm{H}-3), 8.01(\mathrm{~d}, 1 \mathrm{H}, \mathrm{J}=7.8 \mathrm{~Hz}, \mathrm{~J}=5.2 \mathrm{~Hz}, \mathrm{Py} \mathrm{H}-6), 7.82$ $(\mathrm{t}, 1 \mathrm{H}, \mathrm{J}=7.6 \mathrm{~Hz}$, Py $\mathrm{H}-4), 7.26(\mathrm{dd}, 1 \mathrm{H}, \mathrm{J}=7.5 \mathrm{~Hz}$, $\mathrm{J}=5.4 \mathrm{~Hz}$, Py H-5), $4.97\left(\mathrm{~s}, 1 \mathrm{H}, \mathrm{NH}_{2}\right), 4.56(\mathrm{~m}, 1 \mathrm{H}$, cyclohexyl H-1), 4.32 (s, 2H, $\left.\mathrm{CH}_{2}-\mathrm{S}\right), 1.26-1.81(\mathrm{~m}, 10 \mathrm{H}$, cyclohexyl H). ${ }^{13} \mathrm{CNMR}$ (DMSO-d $6,100 \mathrm{MHz}$ ): $\delta 164.5$ $(\mathrm{C}=\mathrm{O}), 152.6,146.8,144.6,143.2,138.4,123.3,120.4$, 56.3, 29.8, 29.2 (2C), 25.4 (2C), 24.9. Anal. Calcd. For $\mathrm{C}_{15} \mathrm{H}_{20} \mathrm{~N}_{6} \mathrm{OS}:$ C, 54.21; H, 6.02; N, 25.30 . 
Found: C, 54.06; H, 6.01; N, 25.10.

2-[\{4-Ethyl-5-(pyridine-2-yl)-4H-1,2,4-triazol-3-yl]sulfanyl\}acetohydrazide $(Z E-3 b)$ Yield 76\%, M.P. 147$148{ }^{\circ} \mathrm{C}, \mathrm{R}_{\mathrm{f}} 0.80$ (ethyl acetate: petroleum ether 2:1); IR $(\mathrm{KBr}) \mathrm{cm}^{-1}: 3270(\mathrm{~N}-\mathrm{H}), 2991(\mathrm{C}-\mathrm{H}), 1670(\mathrm{C}=\mathrm{O}$, amide), $1623(\mathrm{C}=\mathrm{N}), 1417(\mathrm{C}=\mathrm{C})$; ${ }^{1} \mathrm{HNMR}$ (DMSO-d ${ }_{6}$, $400 \mathrm{MHz}): \delta 9.47(\mathrm{~s}, 1 \mathrm{H}, \mathrm{NH}), 8.74(\mathrm{~d}, 1 \mathrm{H}, \mathrm{J}=7.7 \mathrm{~Hz}$, Py H-3), 8.03 (d, 1H, J = 7.9 Hz, Py H-6), 7.83 (t, 1H, $\mathrm{J}=7.5 \mathrm{~Hz}$, Py H-4), 7.28 (dd, $1 \mathrm{H}, \mathrm{J}=7.5 \mathrm{~Hz}, \mathrm{~J}=7.8 \mathrm{~Hz}$, Py H-5), 5.25 (s, 2H, NH$)_{2} 4.38$ (s, 2H, $\left.\mathrm{CH}_{2}-\mathrm{S}\right), 4.19$ $\left(\mathrm{q}, 2 \mathrm{H}, \mathrm{J}=6.7 \mathrm{~Hz}, \mathrm{CH}_{2}\right), 1.32\left(\mathrm{t}, 3 \mathrm{H}, \mathrm{J}=6.9 \mathrm{~Hz}, \mathrm{CH}_{3}\right)$. ${ }^{13}$ CNMR (DMSO-d, $100 \mathrm{MHz}$ ): $\delta 164.7(\mathrm{C}=\mathrm{O}), 153.1$, 147.2, 146.6, 145.4, 134.8, 123.7, 121.3, 41.3, 30.5, 12.8. Anal. Calcd. For $\mathrm{C}_{11} \mathrm{H}_{14} \mathrm{~N}_{6} \mathrm{OS}$ : C, 47.48; H, 5.03; N, 30.21. Found: C, 47.50; H, 5.00; N, 30.13.

2-[\{4-(4-Flurophenyl)-5-(pyridine-2-yl)-4H-1,2,4-triazol-3-yl]sulfanyl\}acetohydrazide (ZE-3c) Yield 71\%, M.P. 241-242 ${ }^{\circ} \mathrm{C}, \mathrm{R}_{\mathrm{f}} 0.69$ (ethyl acetate: petroleum ether 2:1); IR $\left.(\mathrm{KBr}) \mathrm{cm}^{-1}: 3234 \mathrm{~N}-\mathrm{H}\right), 2965(\mathrm{C}-\mathrm{H}), 1665$ (C=O, amide), $1627(\mathrm{C}=\mathrm{N}), 1423(\mathrm{C}=\mathrm{C}) ;{ }^{1} \mathrm{H}$ NMR (DMSO-d 6 , $400 \mathrm{MHz}$ ) $\delta 9.91$ (s, 1H, N-H), 8.65 (d, 1H, J = 7.3 Hz Py H-3), 8.04 $(\mathrm{d}, 1 \mathrm{H}, \mathrm{J}=6.7 \mathrm{~Hz}$, Py H-6), 7.81 (t, $1 \mathrm{H}, \mathrm{J}=7.3 \mathrm{~Hz}, \mathrm{Py} \mathrm{H}-4)$, 7.38 (dd, 1H, J = 7.2 Hz, J = 6.6 Hz, Py H-5), 7.22-7.28 (m, 4H, Ar-H), 5.10 (s, 2H, NH $\left.\mathrm{N}_{2}\right), 4.33\left(\mathrm{~s}, 2 \mathrm{H}, \mathrm{CH}_{2}-\mathrm{S}\right) .{ }^{13} \mathrm{CNMR}$ (DMSO-d $\left.\mathrm{d}_{6}, 100 \mathrm{MHz}\right): \delta 165.1(\mathrm{C}=\mathrm{O}), 160.4(\mathrm{C}-\mathrm{F}), 152.8$, 148.6, 147.9, 144.0, 143.7, 136.3, 125.5 (2C), 123.6, 121.7, 115.6 (2C), 30.6. Anal. Calcd. For $\mathrm{C}_{15} \mathrm{H}_{13} \mathrm{~N}_{6} \mathrm{OSF}$ : C, 58.95; $\mathrm{H}$, 6.35; N, 16.18. Found: C, 52.32; H, 3.77; N, 24.41.

\section{Synthesis of 1,2,4-triazolehydrazones ZE-4(a-c)}

Equimolar quantities of respective hydrazide and aromatic aldehydes $(6 \mathrm{mmol})$ were dissolved in ethanol $(50 \mathrm{~mL})$ containing $2-3 \mathrm{~mL}$ of glacial acetic acid. The reaction mixture was refluxed for $2-3 \mathrm{~h}$ until the completion of reaction as monitored by TLC (ethyl acetate: petroleum ether 2:1). After cooling, the reaction mixture was concentrated in vacuo and the solid obtained was recrystallized from ethanol [15].

$N$-[\{(2-Phenyl)methylidene]-2-(4-cyclohexyl-5-(pyridi ne-3-yl)-4H-1,2,4-triazol-3-yl)sulfanyl\}acetohydrazide (ZE-4a) Yield 66\%, M.P. $148-150{ }^{\circ} \mathrm{C}, \mathrm{R}_{\mathrm{f}} 0.76$ (ethyl acetate: petroleum ether 2:1); IR $(\mathrm{KBr}) \mathrm{cm}^{-1}$ : 3390-3215 $(\mathrm{NH}), 2990(\mathrm{C}-\mathrm{H}), 1624(\mathrm{C}=\mathrm{O}$, amide $), 1556(\mathrm{C}=\mathrm{N})$, $1465(\mathrm{C}=\mathrm{C}) ;{ }^{1} \mathrm{H}$ NMR (DMSO-d $\left.6,400 \mathrm{MHz}\right): \delta 9.19(\mathrm{~s}$, $1 \mathrm{H}, \mathrm{N}-\mathrm{H}), 8.74$ (bs, $1 \mathrm{H}, \mathrm{N}=\mathrm{CH}), 8.72(\mathrm{~d}, 1 \mathrm{H}, \mathrm{J}=7.2 \mathrm{~Hz}$, Py H-3), 8.02 (d, $1 \mathrm{H}, \mathrm{J}=6.7 \mathrm{~Hz}$, Py H-6), 7.99 (t, $1 \mathrm{H}$, $\mathrm{J}=7.3 \mathrm{~Hz}, \mathrm{Py} \mathrm{H}-4), 7.94(\mathrm{dd}, 1 \mathrm{H}, \mathrm{J}=7.1 \mathrm{~Hz}, \mathrm{~J}=6.7 \mathrm{~Hz}$, Py H-5), 7.50-756 (m, 4H, Ar-H), 4.22 (m, 1H, cyclohexyl $\mathrm{H}-1), 4.13$ (s, 2H, $\mathrm{CH}_{2}-\mathrm{S}$ ), 1.27-1.81 (m, 10H, cyclohexyl H). ${ }^{13} \mathrm{CNMR}\left(\mathrm{DMSO}_{6}, 100 \mathrm{MHz}\right): \delta 166.4(\mathrm{C}=\mathrm{O})$,
152.3, 148.6, 147.5, 143.7, 141.8, 136.8, 135.6, 129.0, 128.5 (2C), 127.3 (2C), 123.3, 120.5, 56.8, 32.0, 31.1 (2C), 26.0, 25.2 (2C). Anal. Calcd. For $\mathrm{C}_{22} \mathrm{H}_{24} \mathrm{~N}_{6} \mathrm{OS}: \mathrm{C}, 62.85 ; \mathrm{H}$, $5.71 ;$ N, 20.00. Found: C, 62.54; H, 5.65; N, 19.96.

$N-[\{(2-P h e n y l)$ methylidene]-2-(4-ethyl-5-(pyridine-2-yl)-4H -1,2,4-triazol-3-yl)sulfanyl\}acetohydrazide (ZE-4b) Yield 81\%, M.P. $160-162{ }^{\circ} \mathrm{C}, \mathrm{R}_{\mathrm{f}} 0.67$ (ethyl acetate: petroleum ether 2:1); IR (KBr) $\mathrm{cm}^{-1}$ : 3375-3237 (N-H), 2989 (C-H), 1637 ( $\mathrm{C}=\mathrm{O}$, amide), $1575(\mathrm{C}=\mathrm{N}), 1498(\mathrm{C}=\mathrm{C})$; ${ }^{1} \mathrm{H}$ NMR (DMSO-d, $400 \mathrm{MHz}$ ); $\delta 9.31$ (bs, $\left.1 \mathrm{H}, \mathrm{NH}\right), 9.10$ (s, $1 \mathrm{H}, \mathrm{N}=\mathrm{CH}), 8.37$ (d, 1H, J = 6.8 Hz, Py H-3), 8.01 (d, $1 \mathrm{H}, \mathrm{J}=7.5 \mathrm{~Hz}, \mathrm{Py} \mathrm{H}-6), 7.72$ (t, $1 \mathrm{H}, \mathrm{J}=6.8 \mathrm{~Hz}, \mathrm{Py} \mathrm{H}-4)$, $7.58(\mathrm{dd}, 1 \mathrm{H}, \mathrm{J}=6.7 \mathrm{~Hz}, \mathrm{~J}=7.6 \mathrm{~Hz}$, Py H-5), 7.33-7.41 $(\mathrm{m}, 4 \mathrm{H}, \mathrm{Ar}-\mathrm{H}), 4.50$ (q, 2H, J = 6.9 Hz, $\left.\mathrm{CH}_{2}\right), 4.12(\mathrm{~s}, 2 \mathrm{H}$, $\mathrm{CH}_{2}-\mathrm{S}$ ), 1.29 (t, 3H, J = 6.9 Hz, $\mathrm{CH}_{3}$ ). ${ }^{13} \mathrm{CNMR}$ (DMSO-d 6 , $100 \mathrm{MHz}$ ): $\delta 165.8,150.7,148.5,148.3,143.9,141.7,137.3$, 135.6, 128.5, 127.6 (2C), 126.9, 122.3, 120.5, 43.8, 32.1, 12.2. Anal. Calcd. For $\mathrm{C}_{18} \mathrm{H}_{18} \mathrm{~N}_{6} \mathrm{OS}$ : C, 59.01; H, 4.91; N, 22.95 . Found: C, 58.96; H, 4.82; N, 22.63.

$N-[\{(2-P h e n y l) m e t h y l i d e n e]-2-(4-(-f l u r o p h e n y l-5-(p y r i d$ ine-2-yl)-4H-1,2,4-triazole-3-yl)sulfanyl\}acetohydrazide (ZE-4c) Yield 80\%, M.P. $195-198{ }^{\circ} \mathrm{C}, \mathrm{R}_{\mathrm{f}} 0.66$ (ethyl acetate: petroleum ether 2:1); IR $(\mathrm{KBr}) \mathrm{cm}^{-1}$ : 3385$3225(\mathrm{~N}-\mathrm{H}), 2985(\mathrm{C}-\mathrm{H}), 1617$ (C=O, amide), 1590 $(\mathrm{C}=\mathrm{N}), 1469(\mathrm{C}=\mathrm{C}) ;{ }^{1} \mathrm{H}-\mathrm{NMR}\left(\mathrm{DMSO}-\mathrm{d}_{6}, 400 \mathrm{MHz}\right)$ : $\delta 9.35$ (bs, $1 \mathrm{H}, \mathrm{N}-\mathrm{H}), 9.05(\mathrm{~s}, 1 \mathrm{H}, \mathrm{N}=\mathrm{CH}), 8.56(\mathrm{~d}, 1 \mathrm{H}$, $\mathrm{J}=6.8 \mathrm{~Hz}$, Py H-3), 7.91 (t, 4H, J = 7.6 Hz, Py H-6), $7.70(\mathrm{t}, 1 \mathrm{H}, \mathrm{J}=6.9 \mathrm{~Hz}$, Py H-4), $7.48(\mathrm{dd}, 1 \mathrm{H}, \mathrm{J}=7.5 \mathrm{~Hz}$, $\mathrm{J}=6.8 \mathrm{~Hz}$, Py H-5), 7.35-7.41 (m, 4H, Ar-H), 7.02-7.10 (m, 4H, Ar-H), 4.29 (s, 2H, $\mathrm{CH}_{2}-\mathrm{S}$ ). ${ }^{13} \mathrm{CNMR}$ (DMSO$\left.\mathrm{d}_{6}, 100 \mathrm{MHz}\right): \delta 165.4(\mathrm{C}=\mathrm{O}), 160.2(\mathrm{C}-\mathrm{F}), 151.3,148.4$, 148.0, 144.7, 143.7, 142.4, 137.4, 135.6, 128.7, $128.2(2 \mathrm{C})$, 127.8 (2C), 127.0 (2C), 123.3, 120.6, 115.8 (2C), 32.1. Anal. Calcd. For $\mathrm{C}_{22} \mathrm{H}_{17} \mathrm{~N}_{6} \mathrm{OSF}$ : C, 61.11; H, 3.93; N, 19.44. Found: C, 61.01; H, 3.95; N, 19.45.

\section{Synthesis of 1,2,4-triazole sulphonamides ZE-5(a-c)}

To a solution of $0.01 \mathrm{~mol}$ of corresponding hydrazides ZE-3(a-e) in ethanol, $0.01 \mathrm{~mol}$ of potassium carbonate and 0.01 mol of $p$-toluene sulfonyl chloride were added. The mixture was refluxed with stirring for $2-3 \mathrm{~h}$. The progress of the reaction was checked by TLC (Ethyl acetate: Petroleum ether 2:1). After completion of the reaction, the reaction mixture was cooled and filtered. The filtrate was then acidified to $\mathrm{pH}$ of 1-2 with $2 \mathrm{~N}$ hydrochloric acid. The solid product separated was filtered and recrystallized from ethanol [16].

$N$-\{(4-Methylphenyl)sulfonyl]-2-(4-cyclohexyl-5-(pyrid ine-2-yl)-4H-1,2,4-triazol-3yl)sulfanyl\}acetohydrazide 
(ZE-5a) Yield 83\%, M.P. $250-251{ }^{\circ} \mathrm{C}, \mathrm{R}_{\mathrm{f}} 0.58$ (ethyl acetate: petroleum ether $2: 1)$; IR $(\mathrm{KBr}) \mathrm{cm}^{-1}: 3337(\mathrm{~N}-\mathrm{H})$, $2985(\mathrm{C}-\mathrm{H}), 1660$ (C=O, amide), $1568(\mathrm{C}=\mathrm{N}), 1404$ $(\mathrm{C}=\mathrm{C}), 1384(\mathrm{O}=\mathrm{S}=\mathrm{O}) ;{ }^{1} \mathrm{H}$ NMR (DMSO-d $\left.6,400 \mathrm{MHz}\right)$ : $\delta 9.51$ (s, 1H, NH), 8.67 (d, 1H, J = 5.9 Hz, Py H-3), 8.01 $(\mathrm{d}, 1 \mathrm{H}, \mathrm{J}=7.9 \mathrm{~Hz}$, Py H-6), 7.57 (t, 1H, J = 6.0 Hz, Py $\mathrm{H}-4), 7.48$ (dd, 1H, J = 7.8 Hz, J = 6.2 Hz, Py H-5), 7.11$7.13(\mathrm{~m}, 4 \mathrm{H}, \mathrm{Ar}-\mathrm{H}), 4.40$ (m, 1H, cyclohexyl H-1), 4.16 (s, 2H, $\left.\mathrm{CH}_{2}-\mathrm{S}\right), 2.27$ (s, 3H, $\left.\mathrm{ArCH}_{3}\right), 1.21-1.81(\mathrm{~m}, 10 \mathrm{H}$, cyclohexyl H). ${ }^{13} \mathrm{CNMR}$ (DMSO-d $\mathrm{d}_{6}, 100 \mathrm{MHz}$ ): $\delta 167.3$ $(\mathrm{C}=\mathrm{O}), 151.5,148.2,147.7,143.9,1143.2,137.9,137.2$, 129.2 (2C), 128.4 (2C), 123.3, 121.1, 56.8, 32.0, 31.1 (2C), 25.8, 25.1 (2C), 20.9. Anal. Calcd. For $\mathrm{C}_{22} \mathrm{H}_{26} \mathrm{~N}_{6} \mathrm{O}_{3} \mathrm{~S}_{2}: \mathrm{C}$, 54.32; H, 5.34; N, 17.28. Found: C, 54.16; H, 5.36; N, 17.15 .

$N-\{(4-M e t h y l p h e n y l)$ sulfonyl]-2-(4-ethyl-5-(pyridine -2-yl)-4H-1,2,4-triazol-3yl)sulfanyl\}acetohydrazide (ZE-5b) Yield $85 \%$, M.P. $265-266{ }^{\circ} \mathrm{C}, \mathrm{R}_{\mathrm{f}} 0.72$ (ethyl acetate: petroleum ether 2:1); IR $(\mathrm{KBr}) \mathrm{cm}^{-1}$ : $3375(\mathrm{~N}-\mathrm{H})$, $2990(\mathrm{C}-\mathrm{H}), 1670$ ( $\mathrm{C}=\mathrm{O}$, amide), $1456(\mathrm{C}=\mathrm{C}), 1500$ $(\mathrm{C}=\mathrm{N}), 1413(\mathrm{O}=\mathrm{S}=\mathrm{O}) ;{ }^{1} \mathrm{H}$ NMR (DMSO-d $\left.6,400 \mathrm{MHz}\right)$ : $\delta 9.21$ (s, 1H, NH), 8.73 (d, 1H, J = 5.7 Hz, Py H-3), 8.14 (d, 1H, J = 7.6 Hz, Py H-6), 7.97 (t, 1H, J = 5.9 Hz, Py $\mathrm{H}-4), 7.55$ (dd, 1H, J = 7.5 Hz, J = 6.0 Hz, Py H-5), 7.10$7.13(\mathrm{~m}, 4 \mathrm{H}, \mathrm{Ar}-\mathrm{H}), 4.50$ (q, 2H, J = 6.6 Hz, $\mathrm{CH}_{2}$ ), 4.13 (s, $2 \mathrm{H}, \mathrm{CH}_{2}-\mathrm{S}$ ), 2.29 (s, 3H, $\mathrm{ArCH}_{3}$ ), 1.33 (t, 3H, J = 6.8 Hz, $\left.\mathrm{CH}_{3}\right) .{ }^{13} \mathrm{CNMR}$ (DMSO-d, $\left.100 \mathrm{MHz}\right): \delta 166.8(\mathrm{C}=\mathrm{O})$, 160.1 (C-F), 151.8, 148.6, 147.9, 144.0, 143.4, 137.8, 137.1, 129.2 (2C), 128.3 (2C), 122.8, 120.3, 43.7, 32.1, 21.0, 12.6. Anal. Calcd. For $\mathrm{C}_{18} \mathrm{H}_{20} \mathrm{~N}_{6} \mathrm{O}_{3} \mathrm{~S}_{2}: \mathrm{C}, 50.00 ; \mathrm{H}$, 4.62; N, 19.44. Found: C, 50.04; H, 4.56; N, 19.41 .

$N$-\{(4-Methylphenyl)sulfonyl]-2-(4-(4-flurophenyl-5-(pyri dine-2-yl)-4H-1,2,4-triazole-3-yl)sulfanyl\}acetohydrazide $(Z E-5 c)$ Yield 61\%, M.P. $240-242{ }^{\circ} \mathrm{C}, \mathrm{R}_{\mathrm{f}} 0.69$ (ethyl acetate: petroleum ether 2:1); IR $(\mathrm{KBr}) \mathrm{cm}^{-1}$ : $3370(\mathrm{NH})$, $2991(\mathrm{C}-\mathrm{H}), 1675$ (C=O, amide), $1446(\mathrm{C}=\mathrm{C}), 1497$ $(\mathrm{C}=\mathrm{N}), 1408(\mathrm{O}=\mathrm{S}=\mathrm{O}) ;{ }^{1} \mathrm{H}$ NMR $\left(\mathrm{DMSO}-\mathrm{d}_{6}, 400 \mathrm{MHz}\right)$ : $\delta 9.60(\mathrm{~s}, 1 \mathrm{H}, \mathrm{NH}), 8.74(\mathrm{~d}, 1 \mathrm{H}, \mathrm{J}=6.7 \mathrm{~Hz}$, Py H-3), $8.01(\mathrm{~d}, 1 \mathrm{H}, \mathrm{J}=7.6 \mathrm{~Hz}, \mathrm{Py} \mathrm{H}-6), 7.95(\mathrm{t}, 1 \mathrm{H}, \mathrm{J}=6.8 \mathrm{~Hz}$, Py H-4), 7.57 (dd, $1 \mathrm{H}, \mathrm{J}=7.6 \mathrm{~Hz}, \mathrm{~J}=6.9 \mathrm{~Hz}$, Py H-5), 7.48-7.51 (m, 4H, ArH), 7.11-7.13 (m, 4H, ArH), 4.16 (s, 2H, $\mathrm{CH}_{2}-\mathrm{S}$ ), 2.33 (s, 3H, $\mathrm{ArCH}_{3}$ ). ${ }^{13} \mathrm{CNMR}$ (DMSO$\left.\mathrm{d}_{6}, 100 \mathrm{MHz}\right): \delta 166.8(\mathrm{C}=\mathrm{O}), 160.1(\mathrm{C}-\mathrm{F}), 151.8,148.6$, $147.9,144.0,143.4,142.8,137.8,137.1,129.2$ (2C), 128.0 (2C), 126.2 (2C), 122.8, 120.3, 115.4 (2C), 32.1. Anal. Calcd. For $\mathrm{C}_{22} \mathrm{H}_{19} \mathrm{~N}_{6} \mathrm{O}_{3} \mathrm{~S} 2 \mathrm{~F}$ : C, 54.32; H, 3.81; N, 16.86 . Found: C, 54.21; H, 3.80; N, 16.69.

\section{Antiplatelet assay}

Antiplatelet activity was determined by whole blood aggregometry method using three different platelet aggregation inducing agonists namely as, A.A, ADP and collagen [17]. Blood samples from healthy volunteers were obtained in clean plastic tubes containing $3.2 \%$ sodium citrate anticoagulant (9:1) and were tested subsequently for 30 -min to 5 -h. The study was performed at $37{ }^{\circ} \mathrm{C}$ at stirring speed of $1200 \mathrm{rpm}$. As per guidelines of the manufacturer, $500 \mu \mathrm{L}$ of citrated blood was diluted with same volume of normal saline. $30 \mu \mathrm{L}$ of different concentrations $(1,3,10,30,100,300$ and $1000 \mu \mathrm{M})$ of test compounds were added and then warmed at $37{ }^{\circ} \mathrm{C}$ in incubation well of aggregometer for 5-min. After placing electrode, aggregation was induced by various stimulatory agonists, like AA $(1.5 \mathrm{mM})$, ADP $(10 \mu \mathrm{M})$ and collagen $(5 \mu \mathrm{g} / \mathrm{mL})$. Response (platelet aggregation) was recorded up to 6-min as electrical impedance in ohms. From these platelet aggregation values of 3-4 individual experiments, percent mean platelet inhibition was calculated.

\section{Anticoagulant activity Plasma recalcification time (PRT)}

Anticoagulant activity of test compounds was determined by PRT method [18]. The blood samples were obtained from normal healthy volunteers in containers containing $3.8 \%$ sodium citrate $(9: 1)$ to prevent the clotting process. Platelet poor plasma was obtained by centrifuging the blood samples at $3000 \mathrm{rpm}$ for $15-\mathrm{min} .200 \mu \mathrm{L}$ plasma, $100 \mu \mathrm{L}$ of different concentrations $(30,100,300$ and $1000 \mu \mathrm{M}$ ) of ZE-4b, ZE-4c, ZE-5a and ZE-5b and 300 $\mu \mathrm{L}$ of $\mathrm{CaCl}_{2}(25 \mathrm{mM})$ were added together in a clean test tube and incubated in a water bath at $37^{\circ} \mathrm{C}$. The clotting time was recorded using stop watch by tilting test tubes every 5-10 s. Heparin $(440 \mu \mathrm{M})$ was used as positive control [19].

\section{Bleeding time (BT)}

Anticoagulant potential of test compounds was also assayed by in vivo tail BT method in mice [20]. Briefly, test compounds ZE-4b, ZE-4c, ZE-5a and ZE-5b in 100, 300 and $1000 \mu \mathrm{g} / \mathrm{kg}$ doses were injected intravenously into the tail vein of mice, fasted overnight. After 10-min, mice were anesthetized using diethyl ether and $2-3 \mathrm{~mm}$ deep cut was made at their tails. The tail was then immersed into PBS previously warmed to $37^{\circ} \mathrm{C}$. BT was recorded from time when bleeding started to the time when it completely stopped. The recording was made up to $10 \mathrm{~min}$.

\section{Docking studies}

Protein-ligand docking studies were performed with test derivatives ZE-4(b-c) and ZE-5(a-b) using AutoDock software against selected targets of platelet aggregation and blood coagulation. Affinity was determined by the 
E-value or binding energy value $(\mathrm{kcal} / \mathrm{mol})$ of the best pose of the ligand-receptor complex. 3D structures of test compounds were drawn in protein data bank (PDB) format through Biovia Discovery Studio Visualizer client 2016. Test compounds were docked against eleven selected target receptors. Six of them being involved in regulation of platelet aggregation were cyclooxygenase-1 (COX-1), glycoprotein-IIb/IIIa (GPIIb/IIIa), glycoprotein-VI (GP-VI), purino receptor $\mathrm{P}_{2} \mathrm{Y}_{12}$, prostacyclin $\left(\mathrm{PG}-\mathrm{I}_{2}\right.$ ) receptor and protein activated receptor-1 (PAR1) with PDB-IDs: $3 \mathrm{~N} 8 \mathrm{X}, 2 \mathrm{VDM}, 2 \mathrm{G} 17,4 \mathrm{PXZ}, 4 \mathrm{~F} 8 \mathrm{~K}$ and 3VW7 respectively. The target proteins mediating blood coagulation process are antithrombin III (ATIII), factor-X (F-X), factor-II (F-II), factor-IX (F-IX) and vitamin-K epoxide reductase (VKOR) having PDB-IDs: 2B4X, 1KSN, 5JZY, 1RFN and 3KP9 respectively. These targets were obtained from http://www.rcsb.org/pdb/ home/home.do in PDB format which were then purified through "Discovery Studio Visualizer" software. Standard drugs were obtained from https://pubchem.ncbi. nlm.nih.gov/search/search.cgi, in mol format and converted to PDB format via Open Babel JUI software. Reference drugs used for platelet receptors include aspirin (PubChem CID: 2244), tirofiban (PubChem CID: 60947), hinokitiol (PubChem CID: 3611), the active metabolite of clopidogrel (PubChem CID: 10066813), beraprost (PubChem CID: 6917951) and vorapaxar (PubChem CID: 10077130). For blood coagulation receptors, standard drugs used were heparin sulfate (PubChem CID: 53477714), apixaban (PubChem CID: 10182969), argatroban (PubChem CID: 92722), pegnivacogin (PubChem CID: 86278323) and warfarin (PubChem CID: 54678486). Discovery Studio Visualizer was also utilized for post-docking analysis and schematic representation of hydrogen bonds (classical and non-classical), hydrophobic interactions and amino acid residues involved in hydrogen bonding of the best-docked pose of the ligand-protein complex.

\section{Statistical analysis}

Data expressed as a mean \pm standard error of mean (SEM) and analyzed by one-way analysis of variance (ANOVA), with post hoc-Tukey's test. $P<0.05$ was considered, as significantly different. The bar graphs were analyzed by Graph Pad Prism (GraphPad, San Diego, CA, USA).

\section{Results}

\section{Chemistry}

The synthesis of all the intermediates and target compounds was accomplished by the reaction sequence shown in Scheme 1. Initially, triazole thioacetate
ZE-2 $(a-c)$ were synthesized by the reaction of corresponding triazoles $\mathrm{ZE}-1(\mathrm{a}-\mathrm{c})$ with ethyl chloroacetate in the presence of $\mathrm{KOH}$, which were converted to hydrazides $\mathrm{ZE}-3(\mathrm{a}-\mathrm{c})$ by reaction with hydrazine hydrate. The treatment of acetohydrazides with benzaldehyde produced the corresponding hydrazone derivatives $\mathrm{ZE}-4(\mathrm{a}-\mathrm{c})$. Also, the intermediate hydrazides were condensed with $p$-toluene sulfonyl chloride to get the sulfonamide derivatives ZE-5 $(\mathrm{a}-\mathrm{c})$. The purity of all the synthesized compounds was established by thin layer chromatography and elemental analysis data. All compounds yielded a single spot in different solvent systems showing the purity of the product. Compounds were further characterized by FTIR, ${ }^{1} \mathrm{HNMR}$ and ${ }^{13} \mathrm{CNMR}$ spectroscopy. The IR spectra of ZE-2(a-c) showed a strong $\mathrm{C}=\mathrm{O}$ stretch of ester at $1728-1732 \mathrm{~cm}^{-1}$. Similarly, ${ }^{1} \mathrm{HNMR}$ and ${ }^{13} \mathrm{CNMR}$ data also confirmed the formation of an ester. A quartet of $\mathrm{CH}_{2}$ at $3.57 \mathrm{ppm}$ and a triplet of $\mathrm{CH}_{3}$ at $1.33 \mathrm{ppm}$ was observed due to ethyl moiety of ester. The methylene protons attached to sulfur appeared downfield at $4.47 \mathrm{ppm}$ as singlet due to deshielding effect of two electron withdrawing groups. Characteristic peaks corresponding to pyridyl moiety were observed downfield in the expected region. The IR spectra of hydrazides ZE-3 $(\mathrm{a}-\mathrm{c})$ showed $\mathrm{NH}$ stretchings at $3234-3347 \mathrm{~cm}^{-1}$ and amide $\mathrm{C}=\mathrm{O}$ appeared at $1665-1687 \mathrm{~cm}^{-1}$ confirming the formation of hydrazides. The ${ }^{1}$ HNMR spectra showed two characteristic absorptions (singlet at 9.25-9.91 ppm and 5.10-5.25 ppm) corresponding to $\mathrm{NH}$ and $\mathrm{NH}_{2}$ protons of hydrazide group. In the ${ }^{1} \mathrm{HNMR}$ spectra of ZE-4(a-c) characteristic singlet at 8.7-9.0 ppm was observed due to $\mathrm{N}=\mathrm{CH}$ of imine moiety. The $\mathrm{NH}$ protons resonated downfield at 8.72-9.57 ppm as a broad singlet. Additional signals due to aromatic protons of phenyl group were observed in the range of 7.23-7.37 ppm as multiplet. The pyridyl protons appeared downfield as expected. The sulfonamide derivatives ZE-5- $(\mathrm{a}-\mathrm{c})$ were also characterized by their IR and NMR data. The IR spectra showed characteristic absorptions due to $\mathrm{O}=\mathrm{S}=\mathrm{O}$ at $1340-1413 \mathrm{~cm}^{-1}$. In the ${ }^{1} \mathrm{HNMR}$ data signals for methyl protons of $p$-toluene sulfonyl moiety were observed as singlet at $2.30 \mathrm{ppm}$. The NH protons appeared downfield as singlets due to deshielding effect of sulfonyl and carbonyl groups. Aromatic protons resonated in the range of 7.33-7.39 ppm. In the ${ }^{13} \mathrm{CNMR}$ spectra of all compounds, carbonyl carbon resonated most downfield at 165-168 ppm and methylene carbon attached to sulfur was observed at $31.2-32.6 \mathrm{ppm}$. Signals corresponding to carbon atoms of triazole moiety were observed at 151-152 and 147-148 ppm. Methine carbon in ZE-4 $(\mathrm{a}-\mathrm{c})$ resonated at $143-144 \mathrm{ppm}$. All the other protons appeared in the expected region. 


\section{Antiplatelet assay \\ Inhibitory effect on AA-induced platelet aggregation}

The antiplatelet activity of compounds ZE-4(b-c) and $\mathrm{ZE}-5(\mathrm{a}-\mathrm{b})$ was determined by whole blood aggregometry method using Chrono-Log impedance aggregometer, model 591. The test compounds were used in 1, 3, $10,30,100,300$ and $1000 \mu \mathrm{M}$ concentrations to observe their inhibitory effect. ZE-4b inhibited platelet aggregation to $4.4 \pm 0.09,8.8 \pm 0.09,30.3 \pm 0.06,41.2 \pm 0.23$, $63.2 \pm 0.06,78 \pm 0.14$ and $89.5 \pm 0.23 \%$ respectively with $\mathrm{IC}_{50}$ value of $40.1 \mu \mathrm{M}$. ZE-4c inhibited platelet aggregation to $7.9 \pm 0.15,15.4 \pm 0.20,29 \pm 0.21,43 \pm 0.18$, $59 \pm 0.03,75 \pm 0.10$ and $86.4 \pm 0.44 \%$ respectively with $\mathrm{IC}_{50}$ value of $55.3 \mu \mathrm{M}$. The antiplatelet effect of ZE-5a was $4.0 \pm 0.12,7.9 \pm 0.06,23.7 \pm 0.15,39.5 \pm 0.21$, $47.4 \pm 0.12,68 \pm 0.35$ and $72.8 \pm 0.59 \%$ respectively with $\mathrm{IC}_{50}$ value of $121.6 \mu \mathrm{M}$. Similarly, ZE-5b inhibited platelet aggregation to $8.8 \pm 0.09,11.4 \pm 0.27,25 \pm 0.21$, $30.7 \pm 0.58,52.2 \pm 0.40,68.4 \pm 0.40$ and $79 \pm 0.60 \%$ respectively with $\mathrm{IC}_{50}$ value of $99.9 \mu \mathrm{M}$. The standard drug aspirin exhibited inhibition of $27.2 \pm 0.18$, $36 \pm 0.09,50.1 \pm 0.16,59.7 \pm 0.09$ and $100 \%$ respectively with $\mathrm{IC}_{50}$ value of $10.01 \mu \mathrm{M}$, as presented in Table 1.

\section{Inhibitory effect on ADP-induced platelet aggregation}

At $1,3,10,30,100,300$ and $1000 \mu \mathrm{M}$ concentrations of the test compounds, ZE-4b inhibited platelet aggregation to $0.1 \pm 0.03,1.0 \pm 0.03,3.6 \pm 0.03,9.6 \pm 0.06$, $18.2 \pm 0.12,39.4 \pm 0.17$ and $54.7 \pm 0.18 \%$ respectively with $\mathrm{IC}_{50}$ value of $785 \mu \mathrm{M}$. ZE-4c inhibited platelet aggregation to $0.1 \pm 0.03,2.7 \pm 0.06,9.6 \pm 0.15,22.5 \pm 0.06$, $32 \pm 0.12,39.7 \pm 0.23$ and $52.8 \pm 0.12 \%$ respectively with $\mathrm{IC}_{50}$ value of $850.4 \mu \mathrm{M}$. The antiplatelet effect of $\mathrm{ZE}-5 \mathrm{a}$ was observed to be $0.1 \pm 0.09,1.8 \pm 0.06,12.2 \pm 0.12$, $24.3 \pm 0.09,28.5 \pm 0.12,36.3 \pm 0.18$ and $50.9 \pm 0.17 \%$ respectively with $\mathrm{IC}_{50}$ value of $956.8 \mu \mathrm{M}$. ZE-5b inhibited platelet aggregation to $1 \pm 0.03,3.6 \pm 0.06,8.7 \pm 0.17$, $22.5 \pm 0.06,37.1 \pm 0.14,44.9 \pm 0.03$ and $61.2 \pm 0.17 \%$ respectively with $\mathrm{IC}_{50}$ value of $519 \mu \mathrm{M}$. Aspirin exhibited inhibition of $3.6 \pm 0.07,6.2 \pm 0.09,19.1 \pm 0.07$, $25 \pm 0.06,32.8 \pm 0.10,49.8 \pm 0.12$ and $56.9 \pm 0.18 \%$ respectively with $\mathrm{IC}_{50}$ value of $308.4 \mu \mathrm{M}$ as presented in Table 1.

\section{Inhibitory effect on collagen-induced platelet aggregation}

The test compounds were evaluated for collagen-induced platelet aggregation inhibition at concentrations of 1 , $3,10,30,100,300$ and $1000 \mu \mathrm{M}$. ZE-4b showed inhibition of $27.1 \pm 0.40,39.2 \pm 0.06,49.7 \pm 0.11,63.7 \pm 0.23$, $85.7 \pm 0.06,43.8 \pm 0.35$ and $20.5 \pm 0.35 \%$ respectively with $\mathrm{IC}_{50}$ value of $10.01 \mu \mathrm{M}$. ZE-4c inhibited platelet aggregation to $33.5 \pm 0.81,42.2 \pm 0.24,50 \pm 0.32$, $58.4 \pm 0.32,68.4 \pm 0.24,80.9 \pm 0.26$ and $85.9 \pm 0.18 \%$ respectively with $\mathrm{IC}_{50}$ value of $10 \mu \mathrm{M}$. ZE-5a inhibited to $23.3 \pm 0.11,37.8 \pm 0.49,43.3 \pm 0.17,49.5 \pm 0.23$, $67.6 \pm 0.58,72.9 \pm 0.46$ and $81.4 \pm 0.11 \%$ respectively with $\mathrm{IC}_{50}$ value of $30.1 \mu \mathrm{M}$. The inhibitory effect of ZE-5b was $21.6 \pm 0.35,23.1 \pm 0.41,43.8 \pm 0.65,51.8 \pm 0.43$, $67.8 \pm 0.52,78.6 \pm 0.31$ and $91.1 \pm 0.67 \%$ respectively with the $\mathrm{IC}_{50}$ value of $29.97 \mu \mathrm{M}$. Aspirin inhibited platelet aggregation to $37.2 \pm 0.14,48.7 \pm 0.14,57.7 \pm 0.20$, $68.6 \pm 0.29,71 \pm 0.23,78.6 \pm 0.23$ and $98.1 \pm 0.11 \%$ respectively with $\mathrm{IC}_{50}$ value of $3.2 \mu \mathrm{M}$ as presented in Table 1.

\section{Anticoagulant assay \\ Effect on PRT}

The synthesized derivatives ZE-4(b-c) and ZE-5(ab) were tested for their anticoagulant effect at different concentrations of 30, 100, 300 and $1000 \mu \mathrm{M}$. ZE-4b increased coagulation time to $81.40 \pm 2.58,118.2 \pm 4.53$, $197.8 \pm 3.17$ and $232.8 \pm 3.41 \mathrm{~s}(\mathrm{P}<0.001$ vs. saline group) respectively. ZE-4c increased coagulation time to $84.2 \pm 1.88,142 \pm 3.51,205.6 \pm 5.37$ and $300.2 \pm 3.48 \mathrm{~s}$ ( $\mathrm{P}<0.001$ vs. saline group) respectively. In case of ZE-5a coagulation time increased to $89.8 \pm 2.35,139.8 \pm 3.93$, $190.2 \pm 3.65$ and $286 \pm 2.98 \mathrm{~s}(\mathrm{P}<0.001$ vs. saline group $)$ respectively. Similarly ZE-5b also increased the coagulation time to $79.2 \pm 2.27,114.2 \pm 5.39,171.4 \pm 5.93$, $207.6 \pm 3.92 \mathrm{~s}(\mathrm{P}<0.001 \mathrm{vs}$. saline group) respectively. Heparin, at $440 \mu \mathrm{M}$ concentration, increased coagulation time to $379.4 \pm 9.18 \mathrm{~s}$ (Fig. 2).

\section{Effect on BT}

The effect of test compounds ZE-4(b-c) and ZE-5(ab) on bleeding time (BT) was studied at dose levels of 100,300 and $1000 \mu \mathrm{M}$. ZE-4b increased BT to $63.25 \pm 1.31,95.25 \pm 2.01$ and $134.5 \pm 3.122 \mathrm{~s}(\mathrm{P}<0.001$ vs. saline group) respectively. ZE-4c increased BT to $90.5 \pm 3.12,112.25 \pm 2.66$ and $145.75 \pm 1.60 \mathrm{~s}(\mathrm{P}<0.001$ vs. saline group) respectively. In case of ZE-5a bleeding time increased to $48.25 \pm 2.92,71.25 \pm 2.56$ and $111.75 \pm 3.04 \mathrm{~s}(\mathrm{P}<0.001$ vs. saline group $)$ respectively. $\mathrm{ZE}-5 \mathrm{~b}$ increased BT to $63.25 \pm 1.65,86.5 \pm 1.04$ and $144 \pm 2.38 \mathrm{~s}(\mathrm{P}<0.001$ vs. saline group) respectively. Heparin, at $30 \mu \mathrm{M}$ dose, increased BT to $170.75 \pm 7.75 \mathrm{~s}$ (Fig. 3).

\section{Docking evaluation}

Test compounds showed variable affinities for different platelet and coagulant targets. Against COX-1, ZE-4b, ZE-4c, ZE-5a, ZE-5b and aspirin showed E-value of $-10.4,-10.6,-10.1,-9.3$ and $-6.1 \mathrm{kcal} / \mathrm{mol}$ respectively. 2D-interaction diagrams showing hydrogen bonds of ZE-4b, ZE-4c, ZE-5a, ZE-5b and aspirin with COX-1 are presented in Fig. 4. ZE-4b, ZE-4c, 
Table 1 Inhibitory effect of $N$-[\{(2-phenyl)methylidene]-2-(4-ethyl-5-(pyridine-2-yl)-4H-1,2,4-triazole-3-yl)sulfanyl\}acetohydrazide (ZE-4b), N-[\{(2-phenyl)methylidene]-2-(4-(fluorophenyl-5-(pyridine-2-yl)-4H-1,2,4-triazole-3yl)sulfanyl $\}$ acetohydrazide (ZE-4c), $\mathrm{N}$-[\{(4-methylphenyl)sulfonyl\}]-2-(4-cyclohexyl-5-(pyridine-2-yl)-4H-1,2,4-triazole-3-yl)sulfanyl\} acetohydrazide (ZE-5a) and $N$-[\{(4-methylphenyl) sulfonyl\}-2-(4-ethyl-5-(pyridine-2-yl)-4H-1,2,4-triazole-3yl)sulfanyl\}acetohydrazide (ZE-5b) on arachidonic acid (AA), adenosine diphosphate (ADP) and collagen induced platelet aggregation

\begin{tabular}{|c|c|c|c|c|c|c|c|c|c|}
\hline \multirow[t]{2}{*}{ Test sample } & \multirow[t]{2}{*}{ Agonists } & \multicolumn{7}{|c|}{$\%$ inhibition of platelet aggregation } & \multirow[t]{2}{*}{$I C_{50}(\mu \mathrm{M})$} \\
\hline & & $1 \mu \mathrm{M}$ & $3 \mu \mathrm{M}$ & $10 \mu \mathrm{M}$ & $30 \mu \mathrm{M}$ & $100 \mu \mathrm{M}$ & $300 \mu \mathrm{M}$ & $1000 \mu \mathrm{M}$ & \\
\hline \multirow[t]{3}{*}{$\mathrm{ZE}-4 \mathrm{~b}$} & AA & $4.4 \pm 0.09$ & $8.8 \pm 0.09$ & $30.3 \pm 0.06$ & $41.2 \pm 0.23$ & $63.2 \pm 0.06$ & $78 \pm 0.14$ & $89.5 \pm 0.23$ & 40.1 \\
\hline & ADP & $0.1 \pm 0.03$ & $1.0 \pm 0.03$ & $3.6 \pm 0.03$ & $9.6 \pm 0.06$ & $18.2 \pm 0.12$ & $39.4 \pm 0.17$ & $54.7 \pm 0.18$ & 785 \\
\hline & Collagen & $27.1 \pm 0.40$ & $39.2 \pm 0.06$ & $49.7 \pm 0.11$ & $63.7 \pm 0.23$ & $85.7 \pm 0.06$ & $43.8 \pm 0.35$ & $20.5 \pm 0.35$ & 10.01 \\
\hline \multirow[t]{3}{*}{$Z E-4 c$} & AA & $7.9 \pm 0.15$ & $15.4 \pm 0.20$ & $29 \pm 0.21$ & $43 \pm 0.18$ & $59 \pm 0.03$ & $75 \pm 0.10$ & $86.4 \pm 0.44$ & 55.3 \\
\hline & ADP & $0.1 \pm 0.03$ & $2.7 \pm 0.06$ & $9.6 \pm 0.15$ & $22.5 \pm 0.06$ & $32 \pm 0.12$ & $39.7 \pm 0.23$ & $52.8 \pm 0.12$ & 850.4 \\
\hline & Collagen & $33.5 \pm 0.81$ & $42.2 \pm 0.24$ & $50 \pm 0.32$ & $58.4 \pm 0.32$ & $68.4 \pm 0.24$ & $80.9 \pm 0.26$ & $85.9 \pm 0.18$ & 10 \\
\hline \multirow[t]{3}{*}{ ZE-5a } & $\mathrm{AA}$ & $4.0 \pm 0.12$ & $7.9 \pm 0.06$ & $23.7 \pm 0.15$ & $39.5 \pm 0.21$ & $47.4 \pm 0.12$ & $68 \pm 0.35$ & $72.8 \pm 0.59$ & 121.6 \\
\hline & ADP & $0.1 \pm 0.09$ & $1.8 \pm 0.06$ & $12.2 \pm 0.12$ & $24.3 \pm 0.09$ & $28.5 \pm 0.12$ & $36.3 \pm 0.18$ & $50.9 \pm 0.17$ & 956.8 \\
\hline & Collagen & $23.3 \pm 0.11$ & $37.8 \pm 0.49$ & $43.3 \pm 0.17$ & $49.5 \pm 0.23$ & $67.6 \pm 0.58$ & $72.9 \pm 0.46$ & $81.4 \pm 0.11$ & 30.1 \\
\hline \multirow[t]{3}{*}{$Z E-5 b$} & $\mathrm{AA}$ & $8.8 \pm 0.09$ & $11.4 \pm 0.27$ & $25 \pm 0.21$ & $30.7 \pm 0.58$ & $52.2 \pm 0.40$ & $68.4 \pm 0.40$ & $79 \pm 0.60$ & 99.9 \\
\hline & ADP & $1 \pm 0.03$ & $3.6 \pm 0.06$ & $8.7 \pm 0.17$ & $22.5 \pm 0.06$ & $37.1 \pm 0.14$ & $44.9 \pm 0.03$ & $61.2 \pm 0.17$ & 519 \\
\hline & Collagen & $21.6 \pm 0.35$ & $23.1 \pm 0.41$ & $43.8 \pm 0.65$ & $51.8 \pm 0.43$ & $67.8 \pm 0.52$ & $78.6 \pm 0.31$ & $91.1 \pm 0.67$ & 29.97 \\
\hline \multirow[t]{3}{*}{ Aspirin } & $\mathrm{AA}$ & $27.2 \pm 0.18$ & $36 \pm 0.09$ & $50.1 \pm 0.16$ & $59.7 \pm 0.09$ & $100 \pm 0$ & $100 \pm 0$ & $100 \pm 0$ & 10.01 \\
\hline & ADP & $3.6 \pm 0.07$ & $6.2 \pm 0.09$ & $19.1 \pm 0.07$ & $25 \pm 0.06$ & $32.8 \pm 0.10$ & $49.8 \pm 0.12$ & $56.9 \pm 0.18$ & 308.4 \\
\hline & Collagen & $37.2 \pm 0.14$ & $48.7 \pm 0.14$ & $57.7 \pm 0.20$ & $68.6 \pm 0.29$ & $71 \pm 0.23$ & $78.6 \pm 0.23$ & $98.1 \pm 0.11$ & 3.2 \\
\hline
\end{tabular}

Values are shown as mean of $\%$ platelet aggregation inhibition $\pm S E M, n=3-4$

ZE-5a, ZE-5b and tirofiban against GP-IIb/IIIa showed E-value of $-8.6,-9.9,-9.9,-8.7$ and $-7.9 \mathrm{kcal} / \mathrm{mol}$ respectively. 2D-interaction showing hydrogen bonds of ZE-4b, ZE-4c, ZE-5a, ZE-5b and tirofiban with GP-IIb/ IIIa receptor are shown in Fig. 5. Against GP-VI, ZE-4b, ZE-4c, ZE-5a, ZE-5b and hinokitiol showed E-value of $-6.4,-7.3,-7.2,-6.9$ and $-5.8 \mathrm{kcal} / \mathrm{mol}$ respectively. Against $\mathrm{P}_{2} \mathrm{Y}_{12}$ receptor, ZE-4b, ZE-4c, ZE-5a, ZE-5b and clopidogrel (active metabolite) showed E-value of - 6.8, - 6.9, - 5.8, - 7.4 and $-8.0 \mathrm{kcal} / \mathrm{mol} \mathrm{respec}-$ tively. Against PG-I $\mathrm{I}_{2}$ receptor, $\mathrm{ZE}-4 \mathrm{~b}, \mathrm{ZE}-4 \mathrm{c}, \mathrm{ZE}-5 \mathrm{a}$, $\mathrm{ZE}-5 \mathrm{~b}$ and beraprost showed E-value of $-6.8,-7.5$, $-8.1,-8.5$ and $-8.3 \mathrm{kcal} / \mathrm{mol}$ respectively. Against PAR-1 receptor, ZE-4b, ZE-4c, ZE-5a, ZE-5b and vorapaxar showed E-value of $-6.5,-7.9,-8.5,-7.7$ and - $12.4 \mathrm{kcal} / \mathrm{mol}$ respectively. Against AT-III receptor, ZE-4b, ZE-4c, ZE-5a, ZE-5b and heparin sulfate showed E-value of $-6.6,-8.1,-8.4,-8.3$ and $-4.1 \mathrm{kcal} / \mathrm{mol}$ respectively. Against F-X, ZE-4b, ZE-4c, ZE-5a, ZE-5b and apixaban showed E-value of $-8.4,-10.1,-8.2$, -8.3 and $-9.2 \mathrm{kcal} / \mathrm{mol}$ respectively. $2 \mathrm{D}$ interaction, showing hydrogen bonds of ZE-4b, ZE-4c, ZE-5a, ZE-5b and apixaban with F-X are shown in Fig. 6. Against F-II, ZE-4b, ZE-4c, ZE-5a, ZE-5b and argatroban showed E-value of $-7.1,-8.0,-7.4,-7.9$ and $-8.0 \mathrm{kcal} / \mathrm{mol}$ respectively. Against F-IX, ZE-4b, ZE-4c, ZE-5a, ZE-5b and pegnivacogin showed E-value of $-8.4,-8.1,-7.2$,
- 7.8 and $-9.6 \mathrm{kcal} / \mathrm{mol}$ respectively. Against VKOR, ZE-4b, ZE-4c, ZE-5a, ZE-5b and warfarin showed E-value of $-7.8,-8.3,-8.3,-7.2$ and $-12.4 \mathrm{kcal} / \mathrm{mol}$ respectively. The best-docked poses of ligand-protein complex, having maximum binding energy values, no of hydrogen bonds (classical and non-classical) and residues involved in hydrogen bonding are summarized in Tables 2 and 3.

\section{Discussion}

A series of six new 1,2,4-triazole derivatives were synthesized by following Scheme 1. Among these were three hydrazone $\mathrm{ZE}-4(\mathrm{a}-\mathrm{c})$ and three sulphonamide derivatives $\mathrm{ZE}-5(\mathrm{a}-\mathrm{c})$. All these were characterized by spectroscopic techniques including FTIR, ${ }^{1} \mathrm{HNMR},{ }^{13} \mathrm{CNMR}$ and elemental analysis data. All the synthesized derivatives were obtained in good yields except ZE-4a and ZE-5c. The compounds obtained in good yields were evaluated for their antiplatelet and anticoagulant potential using different in silico, in vitro and in vivo assays. To assess the antiplatelet potential, three different agonists were used. In AA induced platelet aggregation, test derivatives showed concentration dependent inhibition. The order of test compounds for platelet aggregation inhibition was as ZE-4b $>$ ZE-4c $>$ ZE-5b $>$ ZE-5a. It is also observed that 1,2,4-triazole hydrazone derivatives i.e. ZE-4b and ZE-4c showed better activity than 1,2,4-triazole sulphonamide 


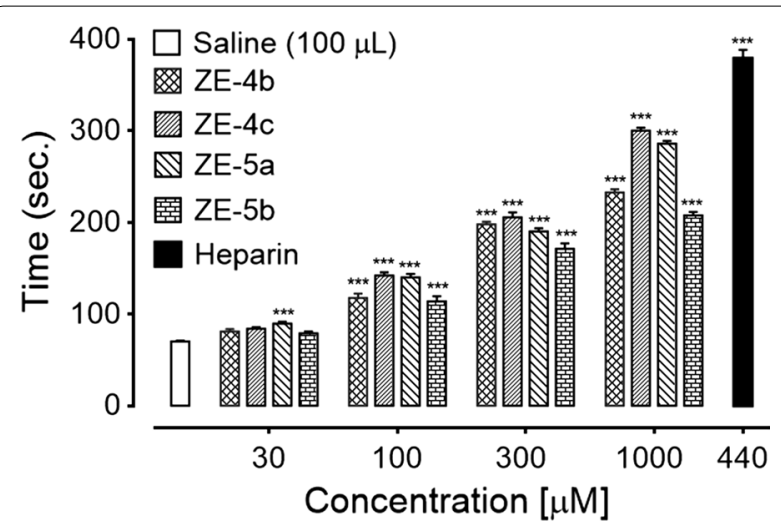

Fig. 2 Bar chart showing increase in plasma recalcification time by different concentrations of $\mathrm{N}$-[\{(2-phenyl)methylidene]-2-(4-ethyl-5(pyridine-2-yl)-4H-1,2,4-triazole-3-yl)sulfanyl\}acetohydrazide (ZE-4b), $\mathrm{N}$-[\{(2-phenyl)methylidene]-2-(4-(fluorophenyl-5-(pyridine-2-yl)-4H1,2,4-triazole-3yl)sulfanyl\}acetohydrazide (ZE-4c), N-[\{(4-methylphenyl)sulfonyl\}]-2-(4-cyclohexyl-5-(pyridine-2-yl)-4H-1,2,4-triazole-3-yl) sulfanyl\}acetohydrazide (ZE-5a), N-[\{(4-methylphenyl)sulfonyl\}-2-(4ethyl-5-(pyridine-2-yl)-4H-1,2,4-triazole-3yl)sulfanyl\} aceto-hydrazide (ZE-5b) and heparin. Data expressed as mean $\pm \mathrm{SEM}, \mathrm{n}=5$, ${ }^{* * *} \mathrm{P}<0.001$ vs. saline group, one way ANOVA with post hoc Tukey's test

derivatives. The possible reason could be the presence of $\mathrm{N}$-acyl hydrazone (NAH) moiety. NAH subunit can increase the antiplatelet potential of compounds because of its high affinity and inhibitory activity for COX-1 resulting in greater inhibition of $\mathrm{TXA}_{2}$ formation [21]. It can also decrease the concentration of intracellular calcium by acting as a calcium chelator and thus can interfere with platelet activation and aggregation [22]. We can infer that ZE-4b and ZE-4c may have inhibited the COX-1 receptor like aspirin, resulting in decreased production of TXA2 and thus inhibition of platelet aggregation [23]. This is also supported by high affinity of test compounds for COX-1. In ADP-induced platelet aggregation, test compounds did not show any significant inhibition, even at a higher dose of $1000 \mu \mathrm{M}$, showing that these derivatives did not interfere significantly with ADP receptors like $\mathrm{P}_{2} \mathrm{Y}_{12}$. In collagen-induced platelet aggregation assay, test compounds exhibited significant inhibition with order of inhibition as ZE-4c $>$ ZE-4b $>$ ZE-5b > ZE-5a. This inhibitory effect clearly indicated the effect of test compounds on collagen receptors i.e. GP-IIb/IIIa or VI [24]. Test compounds have also shown high affinity for GP-IIb/IIIa in docking study, so it is possible that these derivatives interfere the binding of fibrinogen to GP-IIb/IIIa receptor and consequently aggregation of platelets [25]. The synthesized compounds ZE-4(b-c) and ZE-5(a-b) were further investigated for their anticoagulant action via two different models. The test compounds increased PRT and BT with ZE-4c being

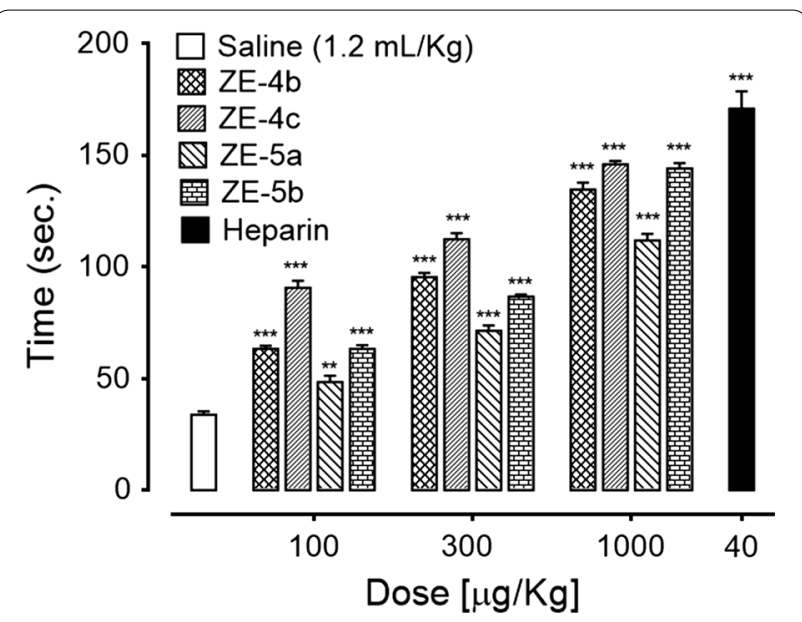

Fig. 3 Bar chart showing increase in tail bleeding time by different doses of $\mathrm{N}$-[\{(2-phenyl)methylidene]-2-(4-ethyl-5-(pyridine-2-yl)4H-1,2,4-triazole-3-yl)sulfanyl\}acetohydrazide (ZE-4b), N-[\{(2-phenyl) methylidene]-2-(4-(fluorophenyl-5-(pyridine-2-yl)-4H-1,2,4-triazole-3yl)sulfanyl\}acetohydrazide (ZE-4C), N-[\{(4-methylphenyl) sulfonyl\}]-2-(4-cyclohexyl-5-(pyridine-2-yl)-4H-1,2,4-triazole-3-yl) sulfanyl\}acetohydrazide (ZE-5a), N-\{(4-methylphenyl)sulfonyl\}-2-(4ethyl-5-(pyridine-2-yl)-4H-1,2,4-triazole-3yl)sulfanyl\}acetohydrazide (ZE-5b) and heparin in mice. Data expressed as mean $\pm S E M, n=4$, ${ }^{* * P}<0.01,{ }^{* *} P<0.001$ vs. saline group, one way ANOVA with post hoc Tukey's test

most effective, which could be attributed to the presence of NAH subunit as it depletes the intracellular calcium by acting as calcium chelator and thus inhibiting the coagulation process [26]. The presence of aromatic $p$-fluorophenyl substitution at $\mathrm{N}-4$ of triazole ring enhanced the anticoagulant effect of ZE-4c [27]. In molecular docking study, ZE-4c have shown high binding energy for F-X.

\section{Conclusions}

In the present study, six new 1,2,4-triazole derivatives $\mathrm{ZE}-4(\mathrm{a}-\mathrm{c})$ and ZE-5(a-c) were synthesized. ZE-4b, ZE-4c, ZE-5a and ZE-5b were obtained in good yield and further evaluated for their antiplatelet and anticoagulant potential. The test compounds showed antiplatelet activity less than the standard drug, however, hydrazone derivatives $\mathrm{ZE}-4 \mathrm{~b}$ and $\mathrm{ZE}-4 \mathrm{c}$ were found to be more potent as compared to sulphonamide derivatives. ZE-4c also exhibited potent anticoagulant activity by increasing PRT and BT time. Further, the molecular interactions of test compounds were investigated by molecular docking studies against selected targets of blood aggregation and coagulation pathways. Test compounds possessed high affinity for COX-1, GP-IIb/IIIa and F-X receptors. The in vitro and in vivo studies also confirmed antiplatelet and anticoagulant potential of test compounds. 


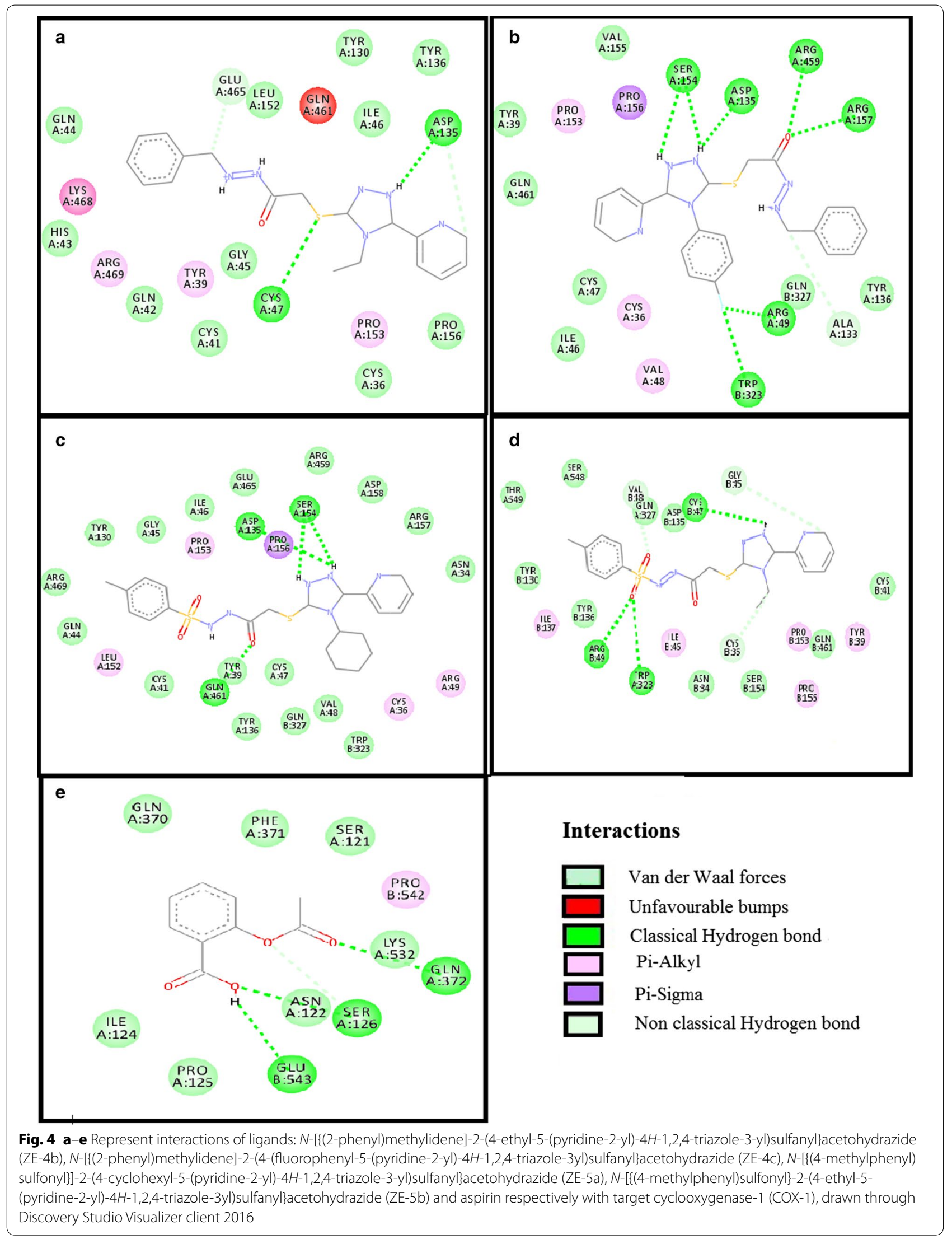




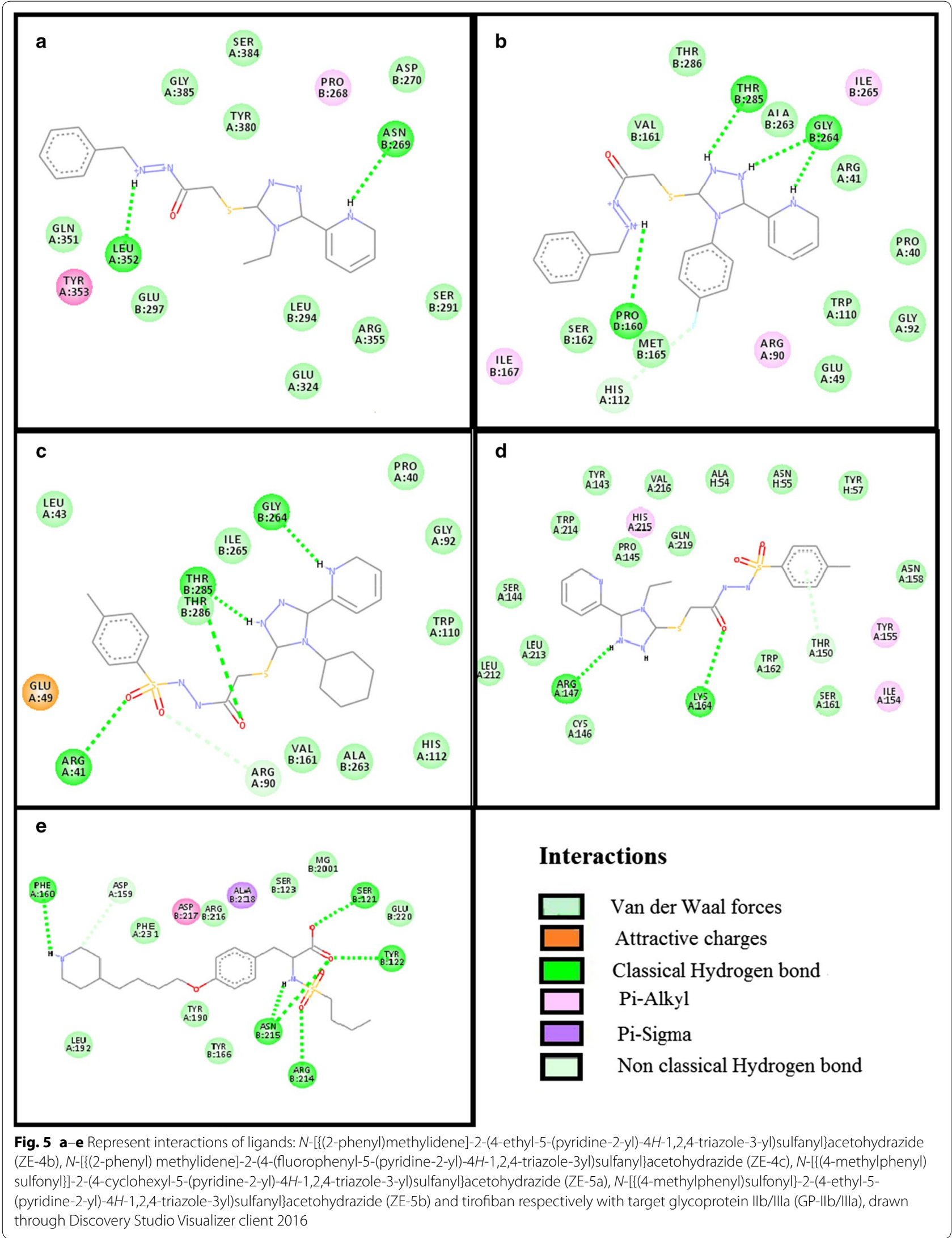




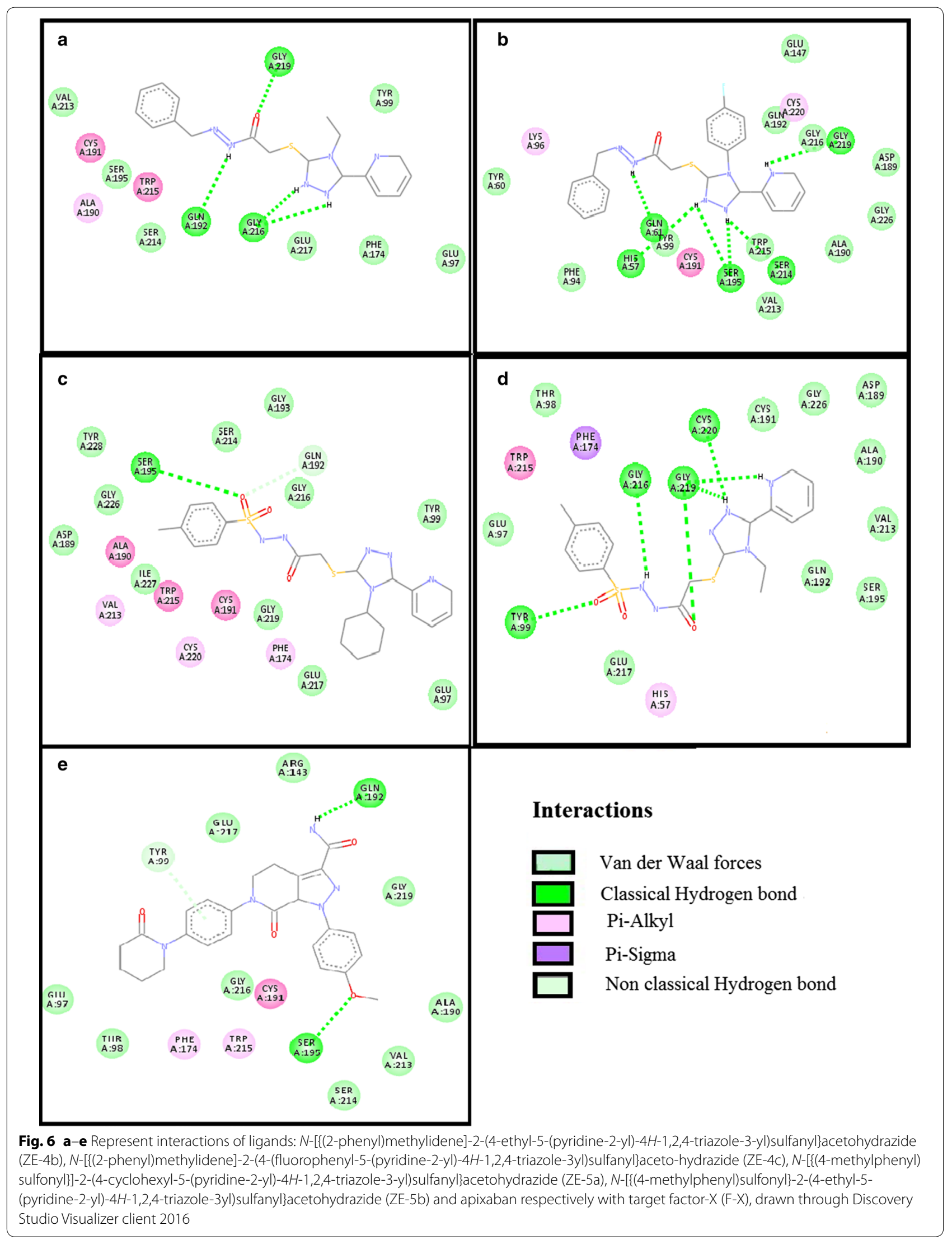




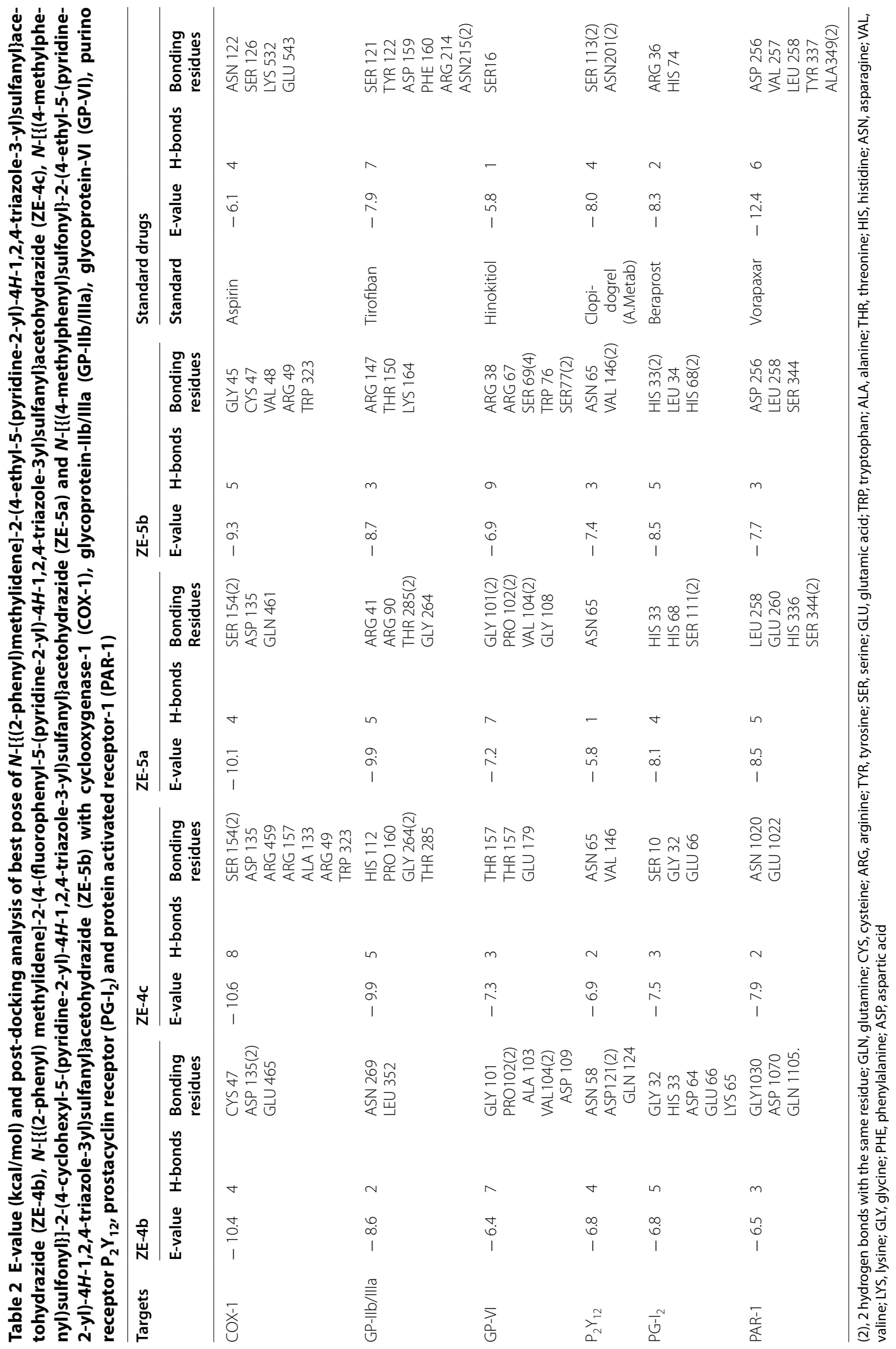




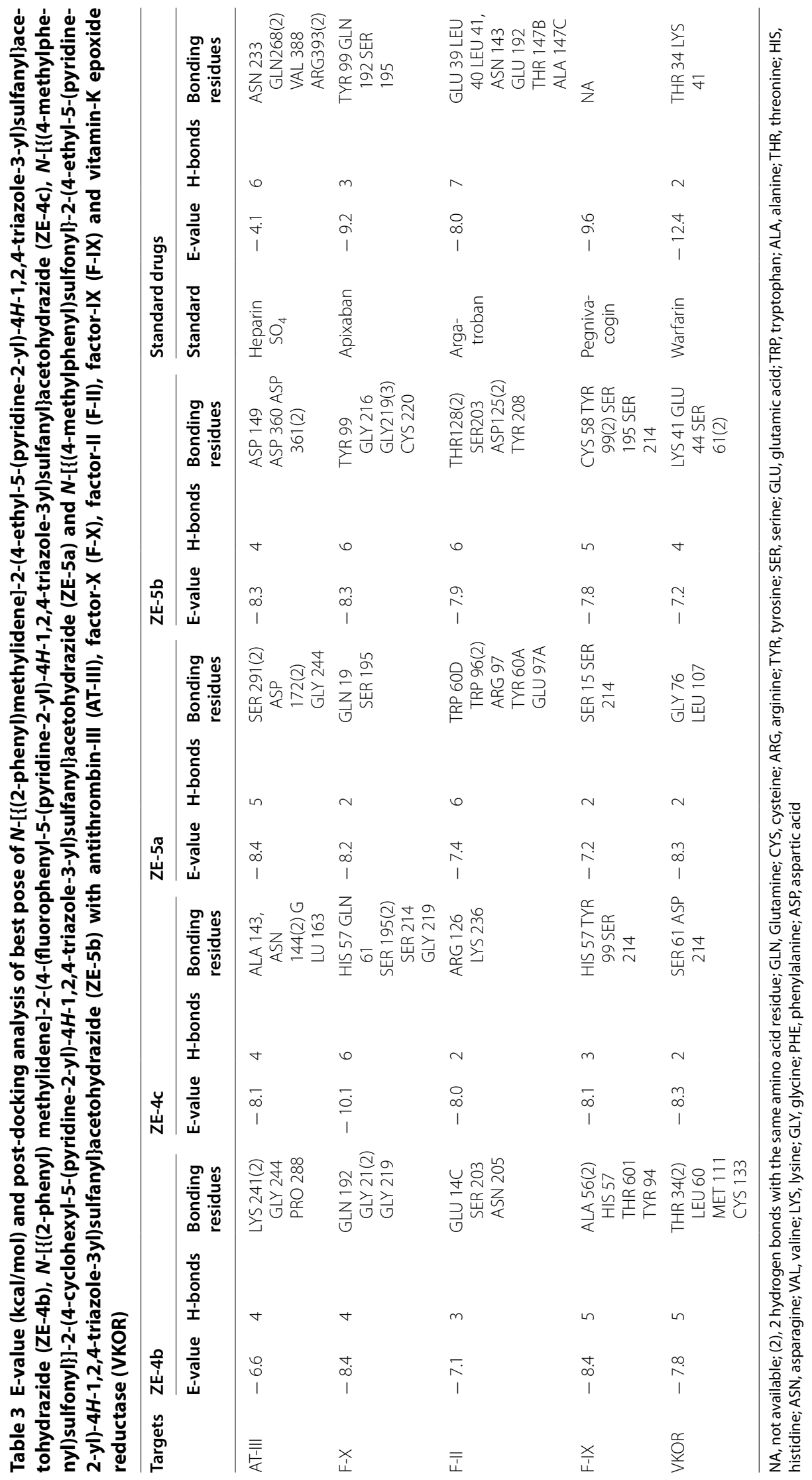




\section{Abbreviations}

ADP: adenosine diphosphate; AA: arachidonic acid; COX-1: cyclooxygenase-1; GP-IIb/IIla: glycoprotein-IIb/IIla; GP-Vl: glycoprotein-Vl; PAR-1: protein activated receptor-1; AT-III: antithrombin-III; PRT: plasma recalcification time; BT: bleeding time; PDB: protein data bank; TXA2: thromboxane-A2; NAH: N-acyl hydrazone.

\section{Authors' contributions}

Authors $A B$ and $H N$ have synthesized and characterized the compounds. WK, A-uK and SA have carried out computational evaluation, antiplatelet and anticoagulant activities of synthesized compounds. All authors read and approved the final manuscript.

\section{Author details}

${ }^{1}$ Riphah Institute of Pharmaceutical Sciences, Riphah International University, Islamabad, Pakistan. ${ }^{2}$ Shifa College of Pharmaceutical Sciences, Shifa Tameere-Millat University, Islamabad, Pakistan.

\section{Acknowledgements}

Authors are thankful to Riphah Academy of Research and Education, Riphah International University for facilitating and partial financial support of the study.

\section{Competing interests}

The authors declare that they have no competing interests.

\section{Availability of data and materials}

All the relevant data supporting the conclusions of this article is included in the article.

\section{Consent for publication}

Written informed consent was obtained from volunteers for the publication of this report and any accompanying images.

\section{Ethics approval and consent to participate}

Consent was obtained from all volunteers. The study was carried out after approval of Research and Ethics Committe.

\section{Funding}

Not applicable. (No specific funding or grant).

\section{Publisher's Note}

Springer Nature remains neutral with regard to jurisdictional claims in published maps and institutional affiliations.

Received: 21 September 2017 Accepted: 23 January 2018

Published online: 07 February 2018

\section{References}

1. Lengauer T, Rarey M (1996) Computational methods for biomolecular docking. Curr Opin Struct Biol 6(3):402-406

2. Horton R (2013) GBD 2010: understanding disease, injury, and risk. Lancet 380(9859):2053-2054

3. Bluestein D (2004) Research approaches for studying flow-induced thromboembolic complications in blood recirculating devices. Expert Rev Med Devices 1(1):65-80

4. Dahlbäck B (2005) Blood coagulation and its regulation by anticoagulant pathways: genetic pathogenesis of bleeding and thrombotic diseases. J Intern Med 257(3):209-223

5. Davie EW, Fujikawa K, Kisiel W (1991) The coagulation cascade: initiation, maintenance, and regulation. Biochemistry 30(43):10363-10370

6. Gangaraju S, Manjappa B, Subbaiah GK, Kempaiah K, Shinde M, Sannaningaiah D (2015) Anticoagulant and antiplatelet activities of jackfruit (Artocarpus heterophyllus) seed extract. Int J Pharm Pharm Sci 7(13):187-191
7. Schlitt A, Rubboli A, Airaksinen KJ, Lip GY (2013) Antiplatelet therapy and anticoagulants. Lancet 382(9886):24-25

8. Asif M (2014) A brief review on anti tubercular activity of pharmacological active some triazole analogues. Global J Res Rev 1(3):51-58

9. Moura A, de Almeida CM, da Silva A, de Souza V, Ferreira V, Menezes M, Kaiser C, Ferreira S, Fuly LA (2016) Synthesis, anticlotting and antiplatelet effects of 1,2,3-triazoles derivatives. Med Chem 12(8):733-741

10. Rollas S, Küçükgüzel SG (2007) Biological activities of hydrazone derivatives. Molecules 12(8):1910-1939

11. Bhat M, Imran M, Khan S, Siddiqui N (2005) Biological activities of sulfonamides. Indian J Pharm Sci 67(2):151-159

12. Akhtar $H$ (2006) Synthesis and characterization of potentially bioactive nucleosides bearing different heterocyclic moieties. PhD thesis. Department of chemistry, Quaid e Azam University, Islamabad

13. Bayrak H, Demirbas A, Karaoglu SA, Demirbas N (2009) Synthesis of some new 1,2,4-triazoles, their Mannich and Schiff bases and evaluation of their antimicrobial activities. Eur J Med Chem 44(3):1057-1066

14. Ahmed M, Sharma R, Nagda DP, Jat JL, Talesara GL (2006) Synthesis and antimicrobial activity of succinimido (2-aryl-4-oxo-3-\{[(quinoline-8-yloxy) acetyl] amino\}-1,3-thiazolidine-5-yl) acetates. Arkivoc 1:66-75

15. Belskaya NP, Dehaen W, Bakulev VA (2010) Synthesis and properties of hydrazones bearing amide, thioamide and amidine functions. Arkivoc 1:275-332

16. Al-Saadi MS, Faidallah HM, Rostom SA (2008) Synthesis and biological evaluation of some 2,4,5-trisubstituted thiazole derivatives as potential antimicrobial and anticancer agents. Arch Pharm Chem Life Sci 341(7):424-434

17. Bauriedel G, Skowasch D, Schneider M, Andrié R, Jabs A, Lüderitz B (2003) Antiplatelet effects of angiotensin-converting enzyme inhibitors compared with aspirin and clopidogrel: a pilot study with whole-blood aggregometry. Am Heart J 145(2):343-348

18. Dey P, Bhakta T (2012) Evaluation of in vitro anticoagulant activity of Molineria recurpata leaf extract. J Nat Prod Plant Resour 2(6):685-688

19. Lee W, Yoo H, Ku S-K, Kim JA, Bae J-S (2013) Anticoagulant activities of piperlonguminine in vitro and in vivo. BMB Rep 46(10):484

20. Dejana E, Callioni A, Quintana A, Gaetano G (1979) Bleeding time in laboratory animals. II-A comparison of different assay conditions in rats. Thromb Res 15(1-2):191-197

21. Todeschini AR, Miranda AL, Silva KC, Parrini SC, Barreiro EJ (1998) Synthesis and evaluation of analgesic, anti-inflammatory and antiplatelet properties of new 2-pyridylaryl hydrazone derivatives. Eur J Med Chem 33(3):189-199

22. Fraga AG, Rodrigues CR, Miranda AL, Barreiro EJ, Fraga CA (2000) Synthesis and pharmacological evaluation of novel heterotricyclic acylhydrazone derivatives, designed as PAF antagonists. Eur J Pharm Sci 11(4):285-290

23. Schrör K (1997) Aspirin and platelets: the antiplatelet action of aspirin and its role in thrombosis treatment and prophylaxis. Semin Thromb, Hemost

24. Nuyttens BP, Thijs T, Deckmyn H, Broos K (2011) Platelet adhesion to collagen. Thromb Res 127:S26-S29

25. Wagner CL, Mascelli M, Neblock D, Weisman H, Coller B, Jordan R (1996) Analysis of GP-IIb/IIla receptor number by quantification of $7 \mathrm{E} 3$ binding to human platelets. Blood 88(3):907-914

26. Chelucci RC, Dutra LA, Lopes Pires ME, Melo TRF, Bosquesi PL, Chung MC, dos Santos JL (2014) Antiplatelet and antithrombotic activities of non-steroidal anti-inflammatory drugs containing an $\mathrm{N}$-acyl hydrazone subunit. Molecules 19(2):2089-2099

27. Parlow JJ, Stevens AM, Stegeman RA, Stallings WC, Kurumbail RG, South MS (2003) Synthesis and crystal structures of substituted benzenes and benzoquinones as tissue factor VIla inhibitors. J Med Chem 46(20):4297-4312 